\title{
Christa Bertelsmeier-Kierst
}

\section{Durchbruch zur Prosa und der Einfluss des Buchdrucks auf die deutschsprachige Erzählliteratur des 15. Jahrhunderts}

\begin{abstract}
The stagnation of German verse romance during the fourteenth century was broken around 1400 when new narratives in prose, combining quite disparate narrative and thematic traditions by transferring material from various cultural spheres and epochs, first appeared. Most of the texts translated into German in the fifteenth century were from the Franco-Burgundian narrative tradition. However, in addition historical subjects from Latin literature, Hellenistic romances, humanistic novels and hagiographical material, as well as Middle High German verse romances, all found their way into Early New High German prose. During this literary development a major event in media history took place, the transition from the hand-written to the printed book. This article analyses which works proved to be successful in the new market for books and which remained in the world of manuscripts. In addition, it asks how far the printed book influenced the development of prose romance and compares the manuscript tradition with the printed editions in North and South Germany during the period of incunabula. Whereas the manuscript tradition consisted of a great many regionally varied versions, only one version was extant in printed editions, or, at most, both an Upper and Lower German version existed in print till 1500, whereas Lower German incunabula mostly follow Upper German editions. There is a clear distinction between Upper German incunabula, especially those printed in Augsburg (the main printing place for vernacular literature in the fifteenth century) and books printed in the South West, where the manuscript tradition, strongly influenced by the French-Burgundian style, still played an important role, also as admired models, for the printed books in this region.
\end{abstract}

Nachdem der deutsche Versroman im 14. Jahrhundert stagnierte, setzte erst gegen 1400 eine neue Erzählliteratur in Prosa wieder ein, die aus unterschiedlichen Kulturräumen und Epochen teils sehr heterogene Themen und narrative Traditionen verarbeitete. Die meisten Texte, die im 15. Jh. ins Deutsche übertragen wurden, sind der französisch-burgundischen Kultur entlehnt, hinzu kommen Geschichtsepen aus dem Lateinischen, hellenistische Romane, humanistische Novellen und hagiographische Stoffe sowie Bearbeitungen älterer mittelhochdeutsche Versromane, die ebenfalls in frühneuhochdeutsche Prosa gegossen wurden. Im Laufe dieser literarischen Entwicklung fand ein wichtiges mediengeschichtliches Ereignis statt: der Übergang vom handgeschriebenen zum gedruckten Buch. Dieser Beitrag untersucht, welche Werke Aufnahme auf dem neuen Buchmarkt fanden und welche hingegen in der Manuskriptkultur verblieben. Zum zweiten fragt er danach, welchen Einfluss der Buchdruck auf den Prosaroman nimmt und vergleicht hierzu die handschriftliche Überlieferung mit den gedruckten Ausgaben in Nord- und Süddeutschland während der Inkunabelzeit. Während in der Manuskriptkultur eine große Vielfalt an regional unterschiedlichen

Christa Bertelsmeier-Kierst, Universität Marburg

Ә Open Access. (C) 2019 Christa Bertelsmeier-Kierst, published by De Gruyter. (c))BY-NC-ND This work is licensed under a Creative Commons Attribution-NonCommercial-NoDerivatives 4.0 International License. https://doi.org/10.1515/9783110563016-002 
Fassungen existiert, setzt sich im Buchdruck in der Regel nur eine Fassung durch oder es werden je eine oberdeutsche und eine niederdeutsche Fassung bis 1500 gedruckt, wobei die niederdeutschen Ausgaben meistens von den oberdeutschen Drucken abhängig sind. Bei den oberdeutschen Inkunabeln lässt sich eine deutliche Differenzierung zwischen den vor allem in Augsburg (Hauptort der deutschsprachigen Druckproduktion im 15. Jh.) hergestellten Büchern und den Drucken aus dem deutschsprachigen Südwesten nachweisen, wo die Manuskriptkultur, stark von der französisch-burgundischen Tradition beeinflusst, nach wie vor eine bedeutende Rolle spielte und auch noch Vorbildfunktion für das gedruckte Buch in dieser Region hatte.

\section{Einleitung}

Obwohl mittlerweile eine breite Diskussion über Fragen der narrativen Kommunikation, über Fiktionalität und Poetik im Mittelalter und in der Frühen Neuzeit stattfindet, existiert bislang keine systematische Erforschung der deutschsprachigen Erzählliteratur. Dies gilt vor allem für den Übergang vom Vers zur Prosa, der sich in Deutschland, von Einzelfällen abgesehen, erst am Ausgang des Mittelalters vollzieht. Während im französisch-angiovinischen Kulturraum bereits im 13. Jahrhundert die älteren Versepen durch Prosafassungen abgelöst werden, setzt der Durchbruch zur Prosa in der erzählenden Literatur in Deutschland erst mit der Wende zum 15. Jahrhundert ein. ${ }^{1}$ Begleitet wird dieser Wandel ab der Mitte des 15. Jahrhunderts von einem wichtigen mediengeschichtlichen Ereignis, der Einführung des Buchdrucks, der schon bald einen bedeutenden Einfluss auf die Entwicklung der volkssprachlichen Literatur nimmt. Während für die Kleinepik, insbesondere für die neue Form der Prosanovellistik, mittlerweile eine Reihe von Gattungsuntersuchungen und Einzelstudien vorliegen, ${ }^{2}$ fehlen

1 Vgl. u.a. Johannes Janota, Orientierung durch volkssprachliche Schriftlichkeit (1280/ 90-1380/90). Geschichte der deutschen Literatur von den Anfängen bis zum Beginn der Neuzeit. Hg. v. Joachim Heinzle. Band III.1 Tübingen 2004, S. 208; Thomas Cramer, 'Aspekte des höfischen Romans im 14. Jahrhundert'. In: Zur deutschen Literatur und Sprache des 14. Jahrhunderts. Dubliner Colloquium 1981. Hg. v. Walter Haug, Timothy R. Jackson und Johannes Janota. Heidelberg 1983, S. 208 ff. [Vgl. für den Übergang zur Prosa im niederländischsprachigen Raum den Beitrag von Besamusca und Willaert (Seite 55) und für die Koëxistenz von Vers und Prosa in England den Beitrag von Sánchez-Martí in diesem Band (Seite 143-166.]

2 Vgl. zum Forschungsstand Wolfgang Achnitz, 'Das Feld der literarischen Kleinformen im Mittelalter’. In: Deutsches Literatur-Lexikon: Das Mittelalter, Bd. 5: Epik. Hg v. Wolfgang Achnitz. Berlin, Boston 2013, S. XXVII-XXXVIII. Zur Rezeption italienischer Novellistik vgl. u.a. Ursula Kocher, Boccaccio und die deutsche Novellistik. Formen der Transposition italienischer "novelle” im 15. u. 16. Jahrhundert. Amsterdam 2005; Luisa Rubini Messerli, Boccaccio deutsch. Die Dekameron-Rezeption in der deutschen Literatur (15.-17. Jahrhundert). Amsterdam 2012 
vergleichbare Untersuchungen zum Prosaroman und zur Geschichtsepik, mit denen - nach dem Stagnieren des höfischen Romans - die narrative Großform um 1400 in Deutschland wieder einsetzt. Über ihre Entwicklung sind wir immer noch schlecht unterrichtet, ${ }^{3}$ weil sich bei Untersuchung der Gattung die Aufmerksamkeit in der Vergangenheit fast ausschließlich auf die Druckprosa richtete, während die große Anzahl der in der Manuskriptkultur verbleibenden Werke in der Forschung weitgehend unbeachtet blieb. Somit wirkt bis in die jüngste Gegenwart wissenschaftsgeschichtlich immer noch die ältere ,Volksbuch'-Tradition nach. ${ }^{4}$

Ausgehend von der Materialbasis, die wir als Marburger Forschergruppe in den letzten Jahren zum frühneuhochdeutschen Prosaroman zusammengetragen haben, ${ }^{5}$ möchte ich nachfolgend beispielhaft untersuchen, welchen

(Chloe 45); Christa Bertelsmeier-Kierst, 'Zur Rezeption des lateinischen und volkssprachlichen Boccaccio im deutschen Frühhumanismus'. In: Giovanni Boccaccio in Europa. Studien zu seiner Rezeption in Spätmittelalter und Früher Neuzeit. Hg. v. Achim Aurnhammer, Rainer Stillers. Wiesbaden 2014 (Wolfenbütteler Abh. zur Renaissanceforschung 31), S. 131-154; Christa Bertelsmeier-Kierst, 'Übersetzen im deutschen Frühhumanismus. Ergebnisse des MRFH zur Einbürgerung humanistischer und antiker Autoren bis 1500'. In: Humanistische Antikenübersetzung und frühneuzeitliche Poetik in Deutschland (1450-1620). Hg. v. Regina Töpfer, Johannes Klaus Kipf und Jörg Robert. Berlin, Boston 2017 (Frühe Neuzeit 211), S. 125-150. Sowie die Ergebnisse des MRFH (http://www.mrfh.de).

3 Richtungweisend immer noch Jan-Dirk Müller, 'Volksbuch/Prosaroman im 15./16. Jahrhundert - Perspektiven der Forschung.' In: Internationales Archiv für Sozialgeschichte der Literatur: Sonderheft Forschungsreferate 1 (1985), S. 1-128; ergänzend Jan-Dirk Müller, 'Prosaroman'. In: Reallexikon der deutschen Literaturwissenschaft 3 (2003), S. 174-177; André Schnyder, 'Der deutsche Prosaroman des 15. und 16. Jahrhunderts'. In: Eulenspiegel trifft Melusine. Der frühneuhochdeutsche Prosaroman im Licht neuer Forschungen und Methoden. Hg. v. Catherine Drittenbass und André Schnyder. Amsterdam, New York 2010 (Chloe 42), S. 11-39; André Schnyder, 'Das Corpus der frühneuhochdeutschen Prosaromane: Eine tabellarische Übersicht als Problemaufriss'. In: Eulenspiegel trifft Melusine, S. 545-556; Christa Bertelsmeier-Kierst, 'Erzählen in Prosa. Zur Entwicklung des deutschen Prosaromans bis 1500’. In: ZfdA 143 (2014), S. 141-165.

4 Vgl. Bertelsmeier-Kierst, 'Erzählen in Prosa' (Anm. 3), S. 161.

5 Das Marburger Prosaroman-Corpus umfasst für den älteren Zeitraum bis 150068 Texte, wobei die Grenze zwischen Roman, Geschichtsepik und Legende oftmals unscharf bleibt, so dass die Hybridität sicher zu den auffälligsten Gattungsmerkmalen in der Frühzeit zählt. Hinzu treten noch Grenzfälle zwischen Klein- und Großepik, so vor allem bei Novellen- und Erzählsammlungen, die durch ihre Rahmenhandlung eine Makrostruktur aufweisen und sich somit der Gattung ,Roman` zumindest annähern. Liste des Textcorpus bei Bertelsmeier-Kierst, 'Erzählen in Prosa' (Anm. 3), S. 163-165. 
Einfluss der mediengeschichtliche Wandel auf die Entwicklung des Prosaromans nimmt, welche Texte sich am erfolgreichsten auf dem neuen Buchmarkt durchsetzen bzw. welche noch überwiegend in der Manuskriptkultur verbleiben. Wie erste Fallstudien inzwischen belegen, ${ }^{6}$ ist es keineswegs so, dass im neuen Medium, dem gedruckten Buch, die formal oder inhaltlich innovativsten Werke tradiert werden, sondern gerade die Texte, die Kontinuität verbürgen und oftmals in einer langen mittelalterlichen Tradition stehen, finden eine frühe und langanhaltende Aufnahme im Repertoire der Buchdrucker.

\section{Antike Stoffe}

Nachdem der Versroman im 14. Jahrhundert in Deutschland stagnierte, setzt „an der Wende zum 15. Jahrhundert in auffälliger Parallele zum Versroman des 12. Jahrhunderts" ${ }^{\text {"7 }}$ der Neubeginn in Prosa wiederum mit antiken Stoffen ein. ${ }^{8}$ Am Beginn steht das Elsässische Trojabuch eines südwestdeutschen Anonymus (vor 1386), das den Trojanerkrieg Konrads von Würzburg mit der Historia destructionis Troiae Guidos de Columnis kompiliert, ${ }^{9}$ und die süddeutsche Bearbeitung des Buch von Troja durch Hans Mair (um 1391), die sich ausschließlich auf den mittellateinischen Text stützt. ${ }^{10}$ Ebenfalls noch vor 1400 dürfte Meister Wichwolt seine Cronica Alexander verfasst haben, ${ }^{11}$ der dann um 1450 der

6 Vgl. hierzu Bertelsmeier-Kierst, 'Übersetzen’ (Anm. 2), mit dem Nachweis speziell für Übersetzungen aus der antiken Literatur im deutschen Frühhumanismus.

7 Janota (vgl. Anm. 1), S. 462 f.

8 Parallel setzt sich auch die Versliteratur fort, wie der fast zeitgleich geschriebene Wernigeroder Alexander bezeugt. Vgl. Dieter Welz, 'Der große Alexander'. In: ${ }^{2} V L ~ 3$ (1981), Sp. 281.

9 Karin Schneider, 'Buch von Troja I'. In: ${ }^{2} V L 1$ (1978), Sp. 1100 und 11 (2004), Sp. 300; Volker Zapf, 'Elsässisches Trojabuch'. In: Deutsches Literatur-Lexikon 5 (vgl. Anm. 2), Sp. 1143-1145. [Bearbeitungen der Historia destructionis Troiae finden sich auch anderswo in Westeuropa; in Frankreich, England und den Niederen Landen fand allerdings vor allem die Bearbeitung Raoul Lefèvres Verbreitung; vgl. die Beiträge von Besamusca und Willaert, S. 72-74, sowie De Bruijn, S. 95-101, in diesem Band).]

10 Vgl. Karin Schneider, 'Buch von Troja nach Guido de Columnis'. In: ${ }^{2} V L 1$ (1978), Sp. 1101-1104; Hans-Hugo Steinhoff, 'Mair, Hans von Nördlingen'. In: ${ }^{2} V L 5$ (1985), Sp. 1180-1183; Mike Malm, 'Mair von Nördlingen, Hans'. In: Deutsches Literatur-Lexikon. Das Mittelalter, Bd. 3: Reiseberichte und Geschichtsschreibung. Hg. v. Wolfgang Achnitz. Berlin, Boston 2011, Sp. 475-477.

11 Gisela Kornrumpf, 'Meister Wichwolt'. In: Killy² 12 (2011), S. 367f; Volker Zapf, 'Meister Wichwolt'. In: Deutsches Literatur-Lexikon 3 (vgl. Anm. 10), Sp. 510-514; Peter H. Andersen, 'Wigbolds Alexanderbuch - Eine Pioniertat'. In: Eulenspiegel trifft Melusine (vgl. Anm. 3), S. $183-200$. 
Alexander des Johannes Hartlieb folgt, dem ein außerordentlicher Erfolg beschieden war. ${ }^{12}$

Vorwiegend handelt es sich bei den Prosabearbeitungen antiker Stoffe um einen Kulturtransfer aus der mittellateinischen Literatur, ihre Übersetzer waren Litterati, d. h. sie gehörten der Schicht der lateinisch Gebildeten an. ${ }^{13}$ Allen diesen frühen Bearbeitern ist das Bemühen um Historisierung des Stoffes gemeinsam; der Alexander dient bei Hartlieb und Wichwolt als Fürstenspiegel; ebenfalls in den Prosabearbeitungen vom Buch von Troja ${ }^{14}$ werden Darstellungen von Minne und Rittertum, wie sie zuvor noch Konrads Trojanerkrieg prägten, stark zurückgedrängt und „die historische Faktizität der Erzählung in den Mittelpunkt“" gerückt. ${ }^{15}$

Wie werden die neuen Prosabearbeitungen antiker Stoffe nun im Druckzeitalter aufgenommen? Als erfolgreichster Text unter den neuen Prosabearbeitungen erweist sich Hartliebs Alexander, der sich mit 20 Handschriften und 11 (9) Drucken bereits bis 1500 einer außerordentlichen Beliebtheit erfreute. ${ }^{16}$ Keiner der übrigen Texte kommt auch nur entfernt an diesen Publikumserfolg heran. Von den zahlreichen Trojabuch-Versionen, die im 15. Jahrhundert auf uns gekommen sind, werden nur zwei verlegt: eine oberdeutsche Druckfassung, die auf einer Kompilation des Elsässischen Trojabuchs mit der Bearbeitung der Historia destructionis Troiae durch Hans Mair aus Nördlingen basiert. Sie wird bis 1500 in sieben Inkunabeln (Erstausgabe: Augsburg 1474) überliefert. $^{17}$ Eine

12 Klaus Grubmüller, ‘Hartlieb, Johannes'. In: ${ }^{2} V L ~ 3$ (1981), Sp. 480-496 und 11 (2004), Sp. 589 f.; Werner Röcke, 'Hartlieb, Johannes'. In: Killy² 12 (2011), S. 35 f.

13 Hartlieb war doctor der erzenie und Leibarzt des bayerischen Herzogs, für den er den Alexander nach Guido de Columnis übersetzte. Hans Mair aus Nörtlingen, Ratsherr, Spitalpfleger und auswärtiger Rechtsvertreter der Stadt, war ein gebildeter Jurist, während Wichwolt, wie die Bezeichnung meister und die selbständige Benutzung mehrerer lateinischer Quellen nahelegt, offenbar den Magistertitel erworben hatte.

14 Vgl. Volker Zapf, 'Buch von Troja nach Guido de Columnis'. In: Deutsches Literatur-Lexikon 5 (vgl. Anm. 2), Sp. 1275-1277. Hinzu kommen noch drei Bearbeitungen, die zu großen Teilen auf Konrads Trojanerkrieg basieren.

15 Zapf (Anm. 14).

16 http://www.handschriftencensus.de/werke/1910; GW 883a-890. Zwei Drucke sind nicht nachweisbar.

17 Die Augsburger Drucke fallen durch verschiedene Redaktionen auf. Vgl. Clemens Alfen, Petra Flochler und Elisabeth Lienert, 'Deutsche Trojatexte des 12.-16. Jhs., Repertorium'. In: Die deutsche Trojaliteratur des Mittelalters und der frühen Neuzeit. Materialien und Untersuchungen. Hg. v. Horst Brunner. Wiesbaden 1990 (Wissensliteratur im Mittelalter 3), S. 104-111; GW 7233-7239. Zwei weitere Straßburger Drucke sind Postinkunabeln aus der Zeit nach 1500 (GW6, Sp. 802a = VD16 H 5679 und VD16 H 5680). 
anonyme niederdeutsche Druckfassung der Historia des Guido de Columnis wurde im 15. Jahrhundert zweimal verlegt. ${ }^{18}$

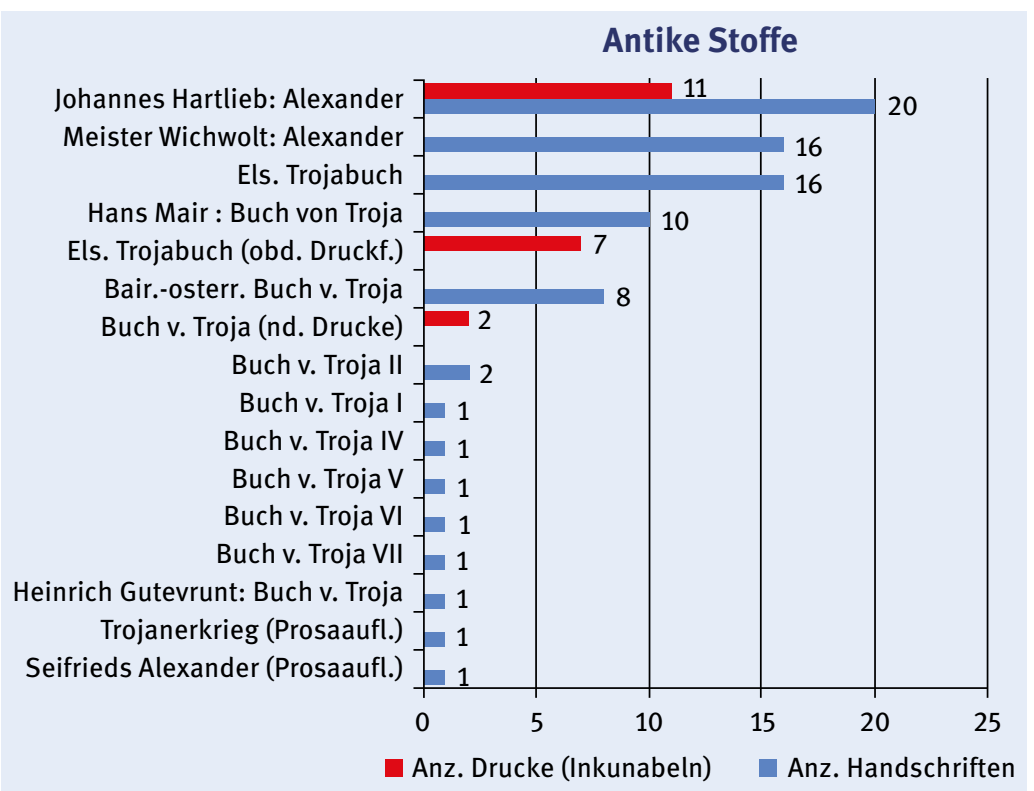

\section{Französisch-burgundische Stoffe}

Neben historisch-antiken Stoffen, die vorzugsweise nach mittellateinischen Quellen bearbeitet wurden, sind es vor allem französische Werke, die als Vorlage frühneuhochdeutscher Prosabearbeitungen dienten. Am Beginn stehen auch hier wieder ,historisch verbürgte‘ Stoffe, nämlich ältere Texte der Chanson de Geste, deren Helden dem Sagenkreis Karls d. Gr. angehören. Ebenfalls die zahlreichen Entlehnungen aus der jüngeren französischen Erzählliteratur lassen bevorzugt Themen des ritterlichen Heidenkampfes und der Kreuzzugsthematik erkennen. Abgesehen von einer niederdeutschen Bearbeitung von Gerart von Rossiliun, die allerdings nur fragmentarisch in einer ostfälischen Handschrift (um 1400)

18 [Lübeck: Lukas Brandis], 1477/78 (GW 7240); [Magdeburg: Moritz Brandis], 1495 (GW 7242). 
erhalten blieb, ${ }^{19}$ setzt der Prosaroman ab den dreißiger Jahren des 15. Jahrhunderts zunächst mit Bearbeitungen der Gräfin Elisabeth von Nassau-Saarbrücken ein, die zu einem eng mit Frankreich verbundenen höfischen Kulturraum zählt. ${ }^{20}$ Offenbar nach einer in Versen geschriebenen Vorlage ${ }^{21}$ verfasste Elisabeth den Prosazyklus von Herpin, Königin Sibille, Loher und Maller sowie Hug Scheppel, wobei die Texte wahrscheinlich schon in der französischen Handschrift ,in einer genealogisch begründeten zyklischen Einheit vorgelegen haben. “22 Überliefert sind die Romane erst nach dem Tod Elisabeths in reich bebilderten Prachtcodices, von denen allein drei ihr Sohn, Johann III. von Saarbrücken ,anlässlich seines Eintritts in den Ritterorden de Croissant nach 1455“23 in Auftrag gab; ihre Tochter, Margarethe von Rodemachern, besaß die Heidelberger Handschrift 1012 mit Loher und Maller, ${ }^{24}$ Margarethe von Savoyen eine Bilderhandschrift von

19 Hamburg, SUB, Cod. germ. 77 (ehem. Werningerode, Gräfl. Stolbergsche Bibliothek). Wann die Dichtung entstanden ist, bleibt ganz ungewiss.

20 Elisabeths Verfasserschaft gilt als umstritten, in jedem Fall sind die Romane aber in ihrer engsten Umgebung entstanden. Zu Elisabeth von Nassau-Saarbrücken und ihrem Kulturraum vgl. Wolfgang Haubrichs und Hans-Walter Herrmann, unter Mitw. v. Gerhard Sauder (Hg.), Zwischen Deutschland und Frankreich. Elisabeth von Lothringen, Gräfin von Nassau-Saarbrücken. St. Ingbert 2002; Bruno Jahn, 'Elisabeth'. In: Deutsches Literatur-Lexikon 5 (vgl. Anm. 2), Sp. 1494-1505.

21 Von der frz. Vorlage, die Elisabeth von ihrer Mutter, Margarethe von Vaudemont-Joinville, für ihre Übersetzungen besaß, ist einzig zu Lohier et Malart ein Blatt aufgetaucht, das als Aktenumschlag des Nassauischen Staatsarchivs überlebt hat und sich heute in Wiesbaden, HStA, Abt. 1105, Nr. 40 befindet. Farbabb. in Eva Horváth und Hans-Walter Storck (Hg.), Von Rittern, Bürgern und von Gottes Wort. Hamburg 2002 (Schriften aus dem Antiquariat Günther, Hamburg, 2), Nr. 30; Textabdruck und Schwarz-Weiß-Abb. in Ute von Bloh, Kurt Gärtner und Michael Heintze, 'Lohier et Malart - Loher und Maller: Vorschläge zu einer Edition des Epos'. In: Zwischen Deutschland und Frankreich (vgl. Anm. 20), S. 427-458, hier 432 und Abb. 30.

22 Hans-Hugo Steinhoff, 'Elisabeth von Nassau-Saarbrücken'. In: ${ }^{2} V L 2$ (1980), Sp. 482-488; Wolfgang Haubrichs, 'Die vier Prosahistorien Elisabeths - Skizzierung ihres Inhalts'. In: Zwischen Deutschland und Frankreich (vgl. Anm. 20), S. 11-16; Ute von Bloh, Ausgerenkte Ordnung. Vier Prosaepen aus dem Umkreis der Gräfin Elisabeth von Nassau-Saarbrücken: Herzog Herpin, Loher und Maller, Huge Scheppel, Königin Sibille. Tübingen 2002 (MTU 119); Ute von Bloh (Hg.), Loher und Maller. Kritische Edition eines spätmittelalterlichen Prosaepos. Berlin 2013 (TMA 50); Bernd Bastert (Hg.), Herzog Herpin. Kritische Edition eines spätmittelalterlichen Prosaepos. Berlin 2014 (TMA 51). Ute von Bloh und Bernd Bastert, Loher und Maller - Herzog Herpin: Kommentar und Erschließung. Berlin 2017 (TMA 55).

23 Peter Jörg Becker, 'Elisabeth von Nassau-Saarbrücken: Herzog Herpin'. In: Aderlass und Seelentrost. Die Überlieferung deutscher Texte im Spiegel Berliner Handschriften und Inkunabeln. Hg. v. Peter Jörg Becker und Eef Overgaauw. Mainz 2003, S. 135-137, hier 135 (Kat. Nr. 63).

$24 \mathrm{Zu}$ den Handschriften, ihrem Buchschmuck und Besitzern vgl. vor allem die Beiträge von Wolfgang Haubrichs, 'Kurze Forschungsgeschichte zum literarischen Werk Elisabeths'. In: Zwischen Deutschland und Frankreich (vgl. Anm. 20), S. 17-48, hier S. 34-38; Hans-Walther Storck, 'Die handschriftliche Überlieferung der Werke 
Herzog Herpin aus der Werkstatt Ludwig Henfflins. ${ }^{25}$ Von Elisabeths Romanen wurde nur Hug Scheppel noch am Ende der Inkunabelzeit gedruckt, allerdings in einer stark gekürzten Bearbeitung, die als Ein lieplich lesen vnd ein // warhafftige Hystorie $^{26}$ im Jahr 1500 auf den Markt kam. ${ }^{27}$ Mit Ausnahme der Königin Sibille, die nicht in die Druckwerkstätten gelangte, wurden die übrigen Werke im 16. Jahrhundert mehrfach verlegt. ${ }^{28}$

Einen beachtlichen Erfolg erzielte bereits im 15. Jahrhundert der Roman Pontus und Sidonia, dessen Stofftradition auf die ältere anglo-normannischen Chanson Horn et Rimenhild zurückreicht, von Geoffrey de la Tour Landry aber noch vor 1400 in den französischen Roman Ponthus et la belle Sidoyne umgearbeitet wurde. ${ }^{29}$ Auf ihm basieren drei deutschsprachige Prosafassungen, wovon die älteste, stark gekürzte Berner Bearbeitung (C) noch in der Mitte des 15. Jahrhunderts entstanden ist. Während diese sich nur unikal in einer Handschrift des Berner Ratsherrn Kaspar von Mülinen (1481-1538) erhalten hat, ${ }^{30}$

Elisabeths von Nassau-Saarbrücken und die malerische Ausstattung der Handschriften'. In: Zwischen Deutschland und Frankreich (vgl. Anm. 20), S. 591-606; sowie Eva Wolff, 'Die Sprache der Bilder. Bild-Erzählung in den Handschriften der Romane der Elisabeth von Nassau-Saarbrücken'. In: Zwischen Deutschland und Frankreich (vgl. Anm. 20), S. 591-622 (mit Farbabb.); Wolfgang Haubrichs, 'Mahl und Krieg. Die Erzählung der Adelskultur in den Texten und Bildern des Hamburger Huge Scheppel der Elisabeth von Lothringen, Gräfin von Nassau-Saarbrücken'. In: Eulenspiegel trifft Melusine (vgl. Anm. 3), S. 201-216 und Abb. (meist in Farbe) 1-14; Horváth und Storck (vgl. Anm. 21), Farbabb. Nr. 31 und 33.

25 Heidelberg UB, Cpg 152. Vgl. Karin Zimmermann, Die Codices Palatini germanici in der Universitätsbibliothek Heidelberg (Cod. Pal. Germ. 1-181). Wiesbaden 2003, S. 332-333; Maria Effinger und Kerstin Losert (Hg.), Mit schönen figuren. Buchkunst im deutschen Südwesten. Heidelberg 2014, S. $72-74$ (Farbabb. Nr. 38); Digitalisat: http://digi.ub.uni-heidelberg.de/digi lit/cpg152.

26 Titelblatt des Hamburger Exemplars des Erstdrucks abgebildet bei Horváth und Storck (vgl. Anm. 21), Nr. 34.

27 Angefertigt hat sie der ehemalige Saarbrücker Bedienstete und Schreiber Conrad Heyndörffer. Vgl. Haubrichs (vgl. Anm. 24), S. 19.

28 Vgl. Gerhard Sauder, 'Die Rezeption der Prosaromane Elisabeths von Nassau-Saarbrücken Vom "Volksbuch" bis zur Romantik'. In: Zwischen Deutschland und Frankreich (vgl. Anm. 20), S. 569-589 (m. Abb.); ergänzend Jahn (vgl. Anm. 20), Sp 197 f. Zur Straßburger Ausgabe des Huge Scheppel von 1537 (VD16 H 5855) vgl. Effinger und Losert (vgl. Anm. 25), S. 99 f. (Abb. 54).

29 Vgl. Xenia von Ertzdorff, Romane und Novellen des 15. und 16. Jahrhunderts in Deutschland. Darmstadt 1989, S. 72-75; Mario Müller, 'Pontus und Sidonia'. In: Deutsches Literatur-Lexikon 5 (vgl. Anm. 2), Sp. 1575-1598.

30 Bern, Burgerbibl., Ms. Mül. 619, S. 1-85. Vgl. Reinhard Hahn, 'Pontus und Sidonia in der Berner Fassung'. In: Daphnis 32 (2003), S. 289-350; Kristina Streun, 'Pontus und Sidonia (C)'. 
wurde die Bearbeitung (A), die der Gemahlin Sigmunds von Tirol, Eleonore von Schottland, zugewiesen wird, ${ }^{31}$ auch im Druck verbreitet. Erstmals in einer Gothaer Handschrift von 1465 (Cod. Chart. A 590) bezeugt, ist sie die einzige Fassung, die von 1483 bis 1600 mindestens siebzehnmal verlegt wurde. Die B-Fassung, die A nahesteht, jedoch ausschmückender erzählt, wird in fünf Handschriften tradiert, die überwiegend aus Adelsbibliotheken stammen und den Prosaroman teils mit älteren Versepen wie Pleiers Tandareis und Flordibel oder Friedrich von Schwaben, teils mit dem älteren Prosa-Lancelot überliefern. ${ }^{32}$ Eine reich illustrierte Handschrift (Heidelberg, UB, Cpg 142) gab 1474 Margarethe von Savoyen, die Gemahlin Ulrichs von Württemberg, wiederum in der Werkstatt Ludwig Henfflins in Auftrag ${ }^{33}$; darüber hinaus ist für die Bibliothek Mechthilds von der Pfalz durch Püterichs Ehrenbrief (1462) schon früh eine Abschrift von Pontus und Sidonia bezeugt. ${ }^{34}$

Ebenfalls in Bern überträgt 1456 der zur städtischen Führungsschicht zählende Thüring von Ringoltingen die Feengeschichte Melusine in deutsche Prosa, ${ }^{35}$ die im Basler Erstdruck mit den Worten eingeleitet wird: Dis ouentúrlich bůch bewiset [...] von einer frowen genant Melusina [...]/ Do by ma[n] briffen mag/ [...] das die hystory wor vnd an ir selber also ist. ${ }^{36}$ Gewidmet hat Thüring seinen Roman dem Markgrafen Rudolf IV. von Hachberg-Röteln, der

In: ${ }^{2} V L 11$ (2004), Sp. 1259 f.; Müller (vgl. Anm. 29), Sp. 1577-1579 (http://www.handschriften census.de/19648).

31 Zur Verfasserfrage siehe den neusten Forschungsbericht bei Müller (vgl. Anm. 29), insb. Sp. $1582 \mathrm{ff}$.

32 Xenia von Ertzdorff, 'Pontus und Sidonia'. In: ${ }^{2} V L 7$ (1989), Sp. 780-782; Müller (vgl. Anm. 29), Sp. $1577 \mathrm{f}$.

33 Zimmermann (vgl. Anm. 25), S. $313 f$ (Digitalisat: http://digi.ub.uni-heidelberg.de/diglit/ cpg142).

34 Vgl. Volker Zapf, 'Püterich von Reichertshausen'. In: Deutsches Literatur-Lexikon 5 (vgl. Anm. 2), Sp. 1664-1669; Faks. Bayerische Staatsbibliothek, Cgm 9220. Mit einer Einf. v. Klaus Grubmüller und einer Beschr. v. Ulrich Montag. München 1999 (Patrimonia 154). Der Hinweis auf Pontus und Sidonia befindet sich in Str. 99.

35 Jan-Dirk Müller, 'Thüring von Ringoltingen'. In: ${ }^{2} V L 9$ (1995), Sp. 908-914; Mike Malm, 'Thüring von Ringoltingen'. In: Deutsches Literatur-Lexikon 5 (vgl. Anm. 2), Sp. 1598-1604; Thüring von Ringoltingen, Melusine (1456). Nach dem Erstdruck Basel: Richel um 1473-1474 hg. v. André Schnyder in Verb. mit Ursula Rautenberg. Bd. II, Kommentar und Aufsätze. Wiesbaden 2006; Jean-Claude Mühlethaler und André Schnyder (Hg.), 550 Jahre dt. Melusine. Couldrette und Thüring von Ringoltingen. Bern 2008 (Beitr. der wiss. Tagung der Universitäten Bern und Lausanne).

36 Zitiert nach dem Faks. (Ex. Karlsruhe, BLB, St. Peter pap. 23, BL. 1a), in Thüring von Ringoltingen, Melusine (Anm. 35), Bd. 1, S. 5. 
großen Einfluss am burgundischen Hof besaß und somit ein wichtiger politischer Bündnisgenosse Berns in den fünfziger und sechziger Jahren des 15. Jahrhunderts war. Als Vorlage diente dem Berner Ratsherrn und späterem Schultheiß eine von Coudrette für die Grafen von Parthenay um 1400 geschriebene Versfassung, die er jedoch in Prosa umsetzte, um den Wahrheitsgehalt der Geschichte $\mathrm{zu}$ unterstreichen. ${ }^{37}$

Thürings selczene vnd gar wunderliche fremde hystorie, ${ }^{38}$ in deren Mittelpunkt Melusine, die Ahnherrin des Kreuzfahrergeschlechts Lusignan, und die erfolgreichen Heidenkämpfe ihrer Söhne stehen, war ein außergewöhnlicher Erfolg beschieden. Bis 1500 wurde der Roman schon in 17 Handschriften und 11 Drucken verbreitet, denen bis ins 19. Jahrhundert noch mindestens 60 Ausgaben folgten. ${ }^{39}$ Hingegen blieb einer anonymen zeitgenössischen Berner Bearbeitung des französischen Prosaromans Cleomades ein vergleichbarer Erfolg versagt. ${ }^{40}$ Auch die Ystoire du vaillant chevalier Pierre, filz du conte de proivence, et la belle Maguelone wird in Deutschland erst durch die Schöne Magelone Veit Warbecks bekannt, der sie 1524 für den sächsischen Kronprinzen bearbeitete und die ab 1535 in zahlreichen Auflagen des 16. und 17. Jahrhunderts verbreitet wurde. ${ }^{41}$ Eine ältere anonyme Prosafassung, die vielleicht noch im letzten Viertel des 15. Jahrhunderts entstanden ist, blieb hingegen nur

$37 \mathrm{Zu}$ den vielfältigen Beglaubigungsstrategien, denen sich Thüring in der Melusine bedient vgl. demnächst meinen Aufsatz 'Rekontextualisierung des Wissens. Res fictae und res factae im vormodernen Roman am Beispiel von Thürings Melusine’. In: Enzyklopädisches Erzählen und vormoderne Romanpoetik. Hg. v. Mathias Herweg, Johannes Klaus Kipf und Dirk Werle (Wolfenbütteler Forschungen) [im Druck].

38 Thüring von Ringoltingen, Melusine (Anm. 35), Bd. 1, S. 5.

39 Vgl. Martina Backes, Fremde Historien. Untersuchungen zur Überlieferungs- und Rezeptionsgeschichte französischer Erzählstoffe im deutschen Mittelalter. Tübingen 2004 (Hermaea 103), S. 103-112; ergänzend: Tina Terrahe, 'Eine neue Hs. der Melusine Thürings von Ringoltingen'. In: ZfdA 138 (2009), S. 50-52. Eine Dokumentation der gesamten Drucküberlieferung ist durch HansJörg Künast und Ursula Rautenberg in Vorbereitung. Forschungsbericht in: Hans-Jörg Künast und Ursula Rautenberg, 'Typographie und Leseweisen. Überlegungen zu den Melusine-Ausgaben der Frankfurter Druckoffizinen [...]'. In: Eulenspiegel trifft Melusine (vgl. Anm. 3), S. 341-359, hier S. 341-343.

40 Der Roman ist nur fragmentarisch in einer einzigen Handschrift der Berner Burgerbibliothek (Mss. hist. helv. VII. 81) zusammen mit der Berner Chronik und der Chronik Jacob Twingers von Königshofen überliefert. Vgl. Volker Zapf, 'Cleomades'. In: Deutsches LiteraturLexikon 5 (vgl. Anm. 2), Sp. 1573-1575; http://www.handschriftencensus.de/20865.

41 Vgl. Hans-Hugo Steinhoff, 'Magelone‘. In: ${ }^{2} V L 5$ (1985), Sp. 1142-1148; Volker Zapf, 'Warbeck'. In: Deutsches Literatur-Lexikon 5 (vgl. Anm. 2), Sp. 2050-2055. 
unikal in einer Handschrift von 1525 erhalten. ${ }^{42}$ Ebenfalls der Roman Paris und Vienna, ${ }^{43}$ der von der Liebe des vramen riddere Paris zu Vienna, der Tochter des Dauphins, erzählt und in Frankreich, England, den Niederlanden und Italien sehr erfolgreich war, ist nur in einer niederdeutschen Bearbeitung auf uns gekommen, die 1488 von Gerhard Leeu in Antwerpen gedruckt wurde, der ein Jahr zuvor auch die niederländische und französische Fassung herausgebracht hatte. Diesen Drucken sind die 26 Holzschnitte für die niederdeutsche Ausgabe entnommen, die dem sog. „Meister von Harlem“ zugesprochen werden. ${ }^{44}$

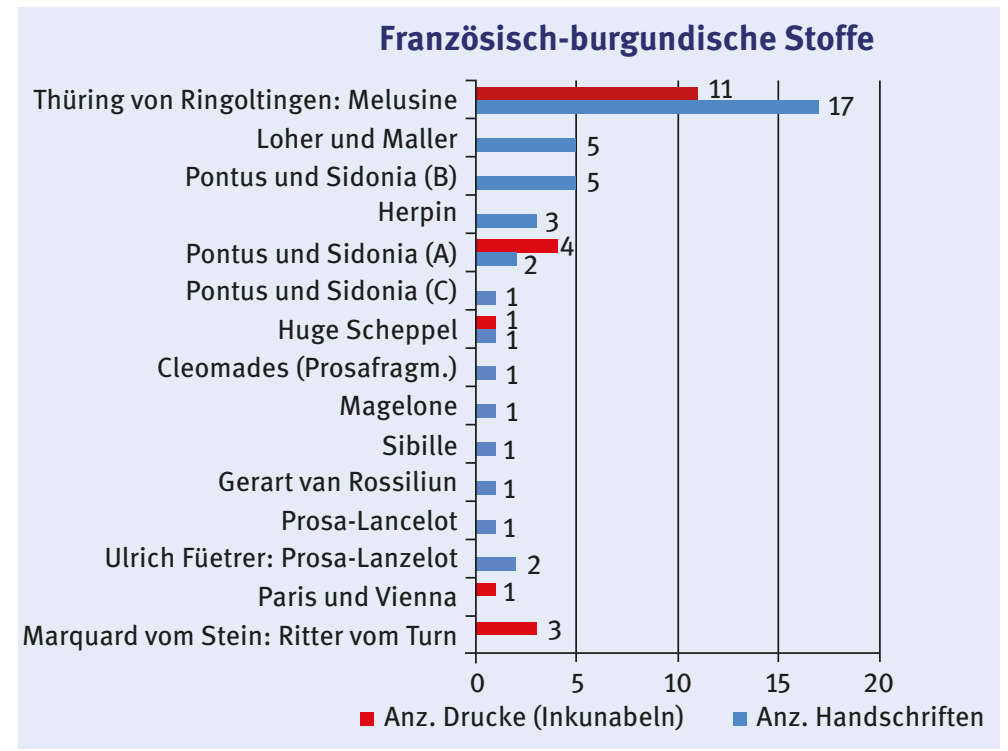

Hingegen war der Erzählsammlung Der Ritter vom Turn, die der württembergische Landvogt von Mömpelgard, Marquart von Stein, nach dem Livre du chevalier de la Tour Landry pour l'enseignement de ses filles im späten 15. Jahrhundert verfasste, ein rascher Erfolg beschieden. Noch drei Inkunabeln tradieren

42 Piro de Prouenze und Magelonna (jetzt: Krakau, BJ, Berol. Mgq 1579). Zum Forschungsstand siehe Steinhoff (vgl. Anm. 41), Sp. 1144; Volker Zapf, 'Magelone'. In: Deutsches LiteraturLexikon 5 (vgl. Anm. 2), Sp. 1881-1884.

43 Ertzdorff (vgl. Anm. 29), S. 62.

44 Jürgen Meier, 'Paris und Vienna'. In: ${ }^{2} V L 7$ (1989), Sp. 306-309; Mike Malm, 'Paris und Vienna'. In: Deutsches Literatur-Lexikon 5 (vgl. Anm. 2), Sp. 1901-1904; GW 12699 (Digitalisat: http://pds.lib.harvard.edu/pds/view/30971723). 
ab 1493 das Werk, das im Basler Erstdruck mit 46 künstlerisch hochwertigen Holzschnitten nach Vorlagen Albrecht Dürers ausgestattet wurde. Im 16. Jahrhundert erlebte der Ritter vom Turn neun weitere Ausgaben. ${ }^{45}$

Wie die meisten deutschen Prosafassungen antiker Stoffe erreichten von den zahlreichen Prosaauflösungen, die der älteren Chanson de Geste und der jüngeren französischen Erzähltradition entlehnt wurden, nur wenige die Druckoffizinen der Inkunabelzeit. Mit Abstand erweist sich hier Thürings Melusine als erfolgreichster Roman, der bis ins 19. Jahrhundert immer wieder verlegt wurde. Verglichen mit den Druckauflagen, die vor allem die Prosabearbeitungen von Werken der jüngeren französischen Erzähltradition erzielten, fällt in Deutschland die große Zurückhaltung gegenüber den altfranzösischen Prosazyklen aus dem Bereich der Artus- und Gralsepik auf, von denen keiner durch den Buchdruck verbreitet wurde. ${ }^{46}$

\section{Mittelhochdeutsche Versepen}

Kommen wir zur dritten bedeutenden Stofftradition, mit der sich der Prosaroman vor 1500 profiliert: die Bearbeitungen älterer mhd. Versepen. Abgesehen von einer nur fragmentarisch erhaltenen ripuarischen Prosafassung des Crane

45 Hans-Joachim Kreutzer, 'Marquart von Stein'. In: ${ }^{2} V L 6$ (1987), Sp. 129-135; Mike Malm, 'Marquart von Stein'. In: Deutsches Literatur-Lexikon 5 (vgl. Anm. 2), Sp. 1925-1929; Anneliese Schmitt, 'La Tour-Landry, Geoffrey de: Der Ritter vom Turm, deutsche Bearbeitung von Marquard Stein'. In: Aderlass und Seelentrost (vgl. Anm. 23), S. 143 f. (Kat. Nr. 66) m. Abb von B1 $3^{\mathrm{r}}$ des Baslers Erstdrucks.

46 Der Prosa-Lancelot, dessen erster Teil fragmentarisch schon um 1250 in Deutschland überliefert ist, wurde offenbar vor 1470 am Heidelberger Hof $(P=$ Cpg 147) fortgesetzt. Neben dem Kurfürsten Friedrich I. käme auch seine Schwester, Mechthild von der Pfalz, als Auftraggeberin in Frage, da sie gemäß Püterichs 'Ehrenbrief' mehrere Lancelot-Handschriften besessen hat (Zimmermann (vgl. Anm. 25), S. 324; Martina Backes, Das literarische Leben am kurpfälzischen Hof zu Heidelberg im 15. Jahrhundert. Ein Beitrag zur Gönnerforschung des Spätmittelalters, Tübingen 1992, S. 168, Anm. 197). Eine nahezu vollständige Bearbeitung, die auch die Lücke in P füllt, erfolgte erst im 16. Jh. Am Münchner Hof verfasste Ulrich Füetrer im 15. Jh. eine kürzende Bearbeitung des Prosa-Lancelot, der er wenig später auch eine strophische Fassung folgen ließ (vgl. Kurt Nyholm, 'Füetrer, Ulrich'. In: ${ }^{2}$ VL 2 (1980), Sp. 999-1007; Mike Malm, 'Fuetrer’. In: Deutsches Literatur-Lexikon 5 (vgl. Anm. 2), Sp. 1867-1873). Noch später ist eine deutsche Bearbeitung des Tristan en prose nachzuweisen. Die wörtliche Übersetzung, die der Gruppe $\mathrm{C}$ der frz. Tradition angehört, ist nur trümmerhaft in einer Handschrift des 16. Jh. auf uns gekommen (vgl. Hans-Hugo Steinhoff, 'Tristan'. In: ${ }^{2} V L 9$ (1995), Sp. 1060 f.; Florian Altenhöfer, 'Tristan'. In: Deutsches Literatur-Lexikon 5 (vgl. Anm. 2), Sp. 1966). [Vgl. für die Verbreitung gedruckter Artusromane in Westeuropa den Beitrag von Montorsi in diesem Band, S. 167-188.] 
Bertolds von Holle ${ }^{47}$ wurden zwei oberdeutsche Prosaromane offenbar unmittelbar für den Buchdruck geschaffen: Tristrant und Isalde nach Eilharts Tristrant (Erstdruck: Augsburg, Anton Sorg, 1484) und Herr Wigoleis vom Rade nach dem Versroman Wirnts von Grafenberg (Erstdruck: Augsburg Johann Schönsperger, d. Ä, 1493). Beide haben eine beachtliche Wirkungsgeschichte bis in die Romantik erfahren und 1807 Joseph Görres zu seiner Schrift Die deutschen Volksbücher veranlasst. Eine stark gekürzte Prosaauflösung des Versromans Wilhelm von Österreich Johanns von Würzburg liegt bis 1500 in zwei Handschriften und zwei Augsburger Drucken von 1481 und 1491 vor. ${ }^{48}$

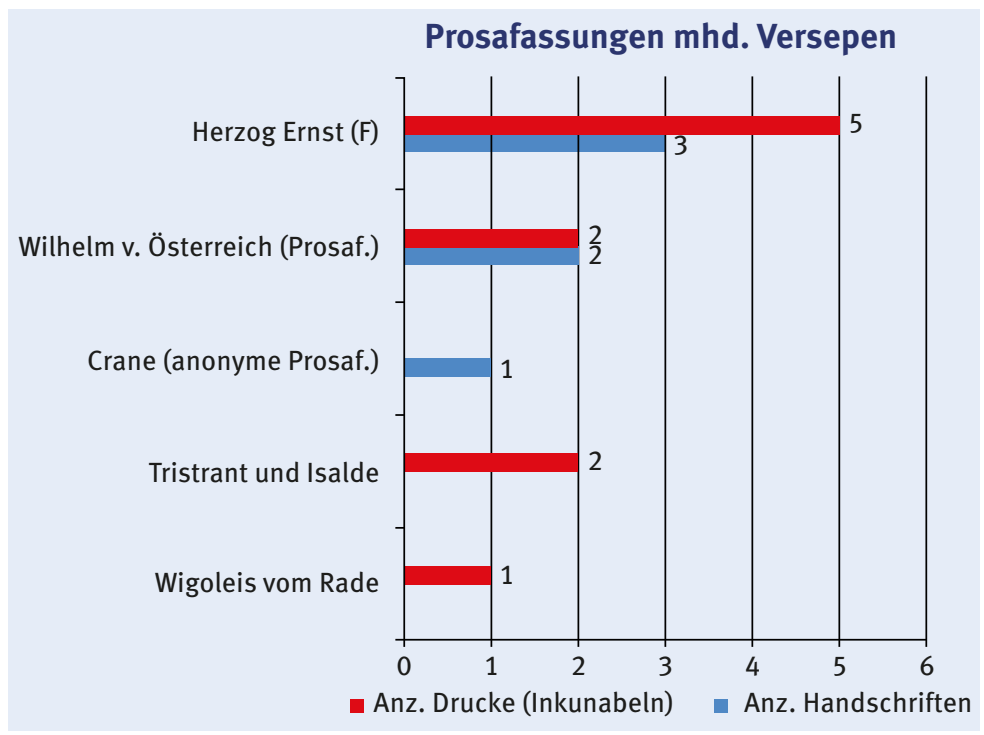

Einen Sonderfall innerhalb der Rezeption mhd. Epen stellt die anonyme Prosabearbeitung des Herzog Ernst (Fassung F) dar, die nicht nach dem mhd. Text,

47 Vgl. Joachim Heinzle, Wandlungen und Neuansätze im 13. Jahrhundert. Geschichte der deutschen Literatur von den Anfängen bis zum Beginn der Neuzeit. Bd. 2, T. 2. Tübingen ${ }^{2} 1994$, S. 113 f. Der Text ist nur unikal überliefert (Darmstadt, LUB, Hs. 2667: Jh.; rip.). Digitalisat: http://tudiget.ulb.tu-darmstadt.de/show/Hs-2667.

48 Vgl. Cora Dietl, 'Wilhelm von Österreich'. In: ${ }^{2} V L 10$ (1999), Sp. 1114-1116; Volker Zapf, 'Wilhelm von Österreich'. In: Deutsches Literatur-Lexikon 5 (vgl. Anm. 2), Sp. 1785-1787. Aus dem 16. Jh. ist nur eine späte Handschrift (Karlsruhe, LB, Cod. Donaueschingen 153) und das Titelblatt eines Druckes (vor 1550) erhalten. 
sondern der lateinischen Bearbeitung des Werks erfolgte. Neben drei Handschriften, von denen zwei den Herzog Ernst mit der Historia Hierosolymitana verbinden, wird der Text seit ca. 1476 auch gedruckt (fünf Inkunabeln und zahlreiche Ausgaben im 16. Jahrhundert). ${ }^{49}$ Textsymbiosen mit Schildbergers Reisebuch oder Brandans Meerfahrt indizieren, dass der Roman vor allem als Reise- und Orientbuch geschätzt wurde. Eine weitere Umarbeitung erfährt der Text dann in den stark gekürzten Ausgaben des 16. Jahrhunderts, die den Roman auf die phantastische Orientreise reduzierten. In dieser Form überlebte der Stoff bis in unsere Tage. ${ }^{50}$

\section{Legenden und Jenseitsvisionen}

Von der Forschung bislang wenig beachtet wurde die große Zahl an Prosabearbeitungen mittelhochdeutscher Verslegenden, religiöser Epen und Visionen. In Prosa übersetzt wurde Der gute Gerhard, wobei der anonyme Bearbeiter die religiöse Argumentation Rudolfs von Ems stark zurücknimmt. ${ }^{51}$ Der Legendenroman von Barlaam und Josaphat liegt gleich in zwei Prosafassungen nach Rudolf von Ems sowie zwei weiteren Bearbeitungen nach lateinischen Vorlagen vor. ${ }^{52}$ Im Zürcher Buch vom hl. Karl wird Strickers Karl mit Konrad Flecks Flore und Blanscheflur verbunden, wobei der stark gekürzte Liebesroman hier die Folie für eine genealogische Vorgeschichte abgibt, bei der die Protagonisten zu Großeltern Karls d. Gr. gemacht werden. ${ }^{53}$

49 Vgl. Volker Zapf, 'Herzog Ernst'. In: Deutsches Literatur-Lexikon 5 (vgl. Anm. 2), Sp. 154 f. und 158; ergänzend: GW 12534, 12535, 12537-12539.

50 Vgl. Peter Hacks, Das Volksbuch von Herzog Ernst, Uraufführung 1967. Zur reichen Rezeptionsgeschichte des Prosa-Herzog Ernst, vgl. Zapf, 'Herzog Ernst' (Anm. 49), S. 154 f.

51 Von Kaiser Otto dem Rotten und dem guoten Gerhard zu Köln. Vgl. Rudolf Benzinger et al. (Hgg.), Der gute Gerhard Rudolf von Ems in einer anonymen Prosaauflösung [...]. Berlin 2001 (DTM 81).

52 Eine obd. und rip. Fassung liegen vor, wobei die obd. Bearbeitung eine vollständige Übersetzung aus dem Speculum historiale des Vincent von Beauvais ist und in einer Handschrift (Berlin, SB, mgq 1147; 150 B11.) sowie zwei illustrierten Augsburger Drucken vor 1500 verbreitet wurde (GW 3398 und 3399). Vgl. Christine Stöllinger-Löser, 'Barlaam und Josaphat'. In: ${ }^{2} V L$ 11 (2004), Sp. 215-219, hier Sp. 216 f.

53 Karl-Ernst Geith, 'Zürcher Buch vom heiligen Karl'. In: ${ }^{2} V L 10$ (1999), Sp. 1597-1600 und 11 (2004), Sp. 1697; Christine Stridde, 'Zürcher Buch vom heiligen Karl'. In: Deutsches LiteraturLexikon 5, Sp. 1781-1783. 
Prosaauflösungen finden sich im 15. Jahrhundert von Hartmanns Gregorius, ${ }^{54}$ Wolframs Willehalm, ${ }^{55}$ dem Georg Reinbots von Durne, ${ }^{56}$ Konrads Alexius, ${ }^{57}$ dem Münchner Oswald ${ }^{58}$ sowie Sente Reinolt (Reinolt von Montalban), der einer mittelniederländischen Vorlage folgte und stofflich eng mit den Haimonskindern verbunden ist, die im 16. Jahrhundert gedruckt wurden. ${ }^{59}$ Mit

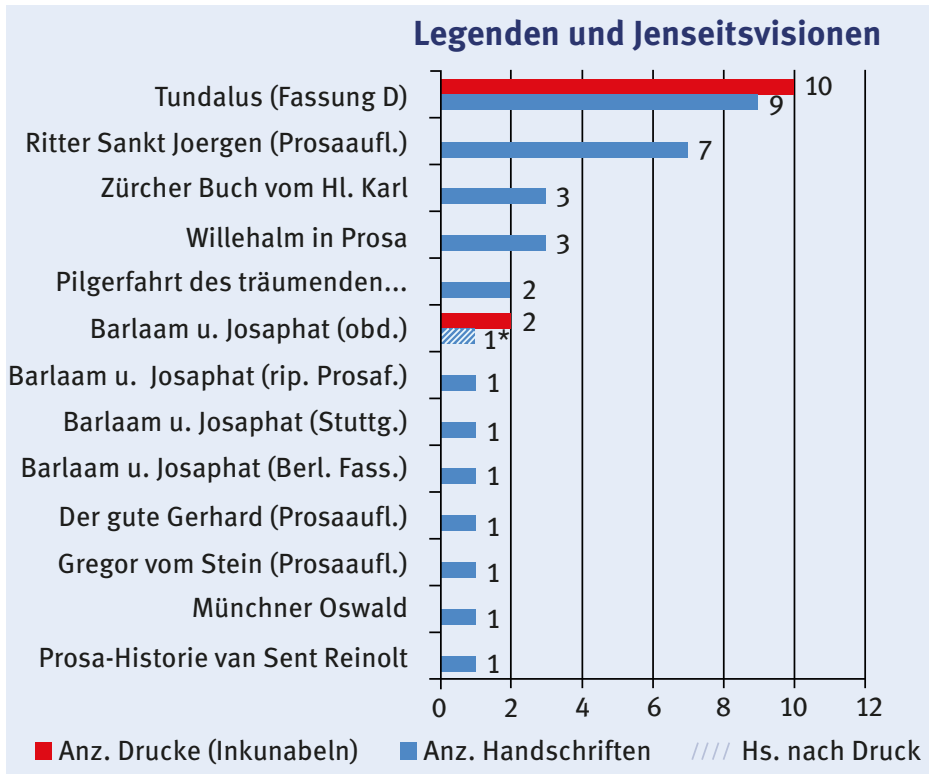

54 Literarische Würdigung der legendarischen Prosaerzählung, insb. auch der Heidelberger Fassung vom Hl. Georg auf dem Stein im Cpg 119, bei Von Ertzdorff (vgl. Anm. 29), S. 10-12; Bernward Plate, Gregorius auf dem Stein, Frühneuhochdeutsche Prosa (15. Jh.) nach dem mittelhochdeutschen Versepos Hartmanns von Aue. Darmstadt 1983 (Texte zur Forschung 39).

55 Holger Deifuß, Hystoria von dem wirdigen ritter sant Wilhelm. Krit. Edition und Untersuchung einer frühneuhochdeutschen Prosauflösung. Frankfurt 2005 (Germ. Arbeiten zu Sprache und Kulturgesch. 45). Vgl. dazu die Rezension von Christoph Gerhardt. In: ZfdA 136 (2007), S. 263-271; Christoph Gerhardt, 'Willehalm (Prosaroman)'. In: ${ }^{2} V L 10$ (1999), Sp. 1151-1154; Konrad Reinhold, 'Willehalm'. In: Deutsches Literatur-Lexikon 5 (vgl. Anm. 2), Sp. 1783 f.

56 Markus Schmitz, Die legent vnd $d z$ leben des hochgelobten manlichen ritters sant joergen. Kritische Neuedition und Interpretation einer alemannischen Prosalegende des heiligen Georg im 15. Jh.. Berlin 2013 (TMA 49); Stridde (vgl. Anm. 53), Sp. 1782.

57 Hans-Friedrich Rosenfeld, 'Alexius [Nachtrag]'. In: ${ }^{2} V L 11$ (2004), Sp. 61-62.

58 Volker Zapf, 'Oswald'. In: Deutsches Literatur-Lexikon 5 (vgl. Anm. 2), Sp. 1260.

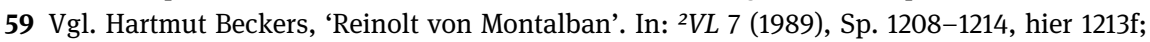
Katharina Philipowski, 'Reinolt von Montelban'. In: Deutsches Literatur-Lexikon 5 (vgl. Anm. 2), Sp. 1605-1608, hier 1606. 
Ausnahme der oberdeutschen Barlaam-Druckfassung, von der sich zwei Inkunabeln erhalten haben, gelangten die übrigen Prosafassungen nicht zum Druck, sondern verblieben in der Manuskriptkultur, soweit sie nicht in stark gekürzten Bearbeitungen in Legendensammlungen integriert und z. B. in Der Heiligen Leben zum Abdruck kamen. ${ }^{60}$

Auf eine außerordentliche Resonanz stieß im Mittelalter die Jenseitsvision vom Ritter Tundalus, die in einer Vielzahl europäischer Versionen verbreitet war. Neben einer frühen Versbearbeitung am Ausgang des 12. Jahrhunderts setzten im 15. Jahrhundert zahlreiche deutschsprachige Prosabearbeitungen des lateinischen Textes ein, von denen hier nur die für ein Laienpublikum geschriebene Fassung D erfasst wurde, die mit neun Handschriften und zehn Inkunabeln ab 1483 ein beachtlicher Erfolg auch im Druckzeitalter beschieden war. ${ }^{61}$

\section{Frühhumanistische Übersetzungen}

Als letzte Gruppe treten in der Phase des deutschen Frühhumanismus vorwiegend Übersetzungen aus der italienischen und antiken Literatur hinzu. ${ }^{62}$ Schwierigkeiten bereitet hier die Abgrenzung von Prosaroman und Novelle, wobei man längere, den Umfang der Novelle deutlich überschreitende Werke wie etwa Enea Silvio Piccolominis Eurialus und Lucretia in Wyles Übersetzung, die bis 1500 in neun Handschriften und fünf Inkunabeln (Erstdruck: Straßburg 1477: 66 Bll.) überliefert ist, ${ }^{63}$ wohl eher als Steinhöwels Griseldis oder Wyles Sigismunda, ${ }^{64}$

60 So wurde z. B. Gregorius auf dem Stein in Der Heiligen Leben gedruckt. Vgl. Klaus Gantert, 'Der Heiligen Leben'. In: Aderlass und Seelentrost (vgl. Anm. 23), S. 218-221 (Kat. Nr. 112) mit Abb. 61 Vgl. Nigel Palmer, 'Tundalus'. In: ${ }^{2} V L 9$ (1995), Sp. 1142-1146, hier 1142 f. (Fassung D); Nigel Palmer, Visio Tnugdali. The German and Dutch Translations and Their Circulation in the Later Middle Ages. München 1982 (MTU 76). Fassung D: Hss. (S. 71 f.) und Drucke (S. 278 ff.); Christine Stridde, 'Tundalus'. In: Deutsches Literatur-Lexikon. Das Mittelalter, Bd. 2: Das geistliche Schrifttum des Spätmittelalters. Hg. v. Wolfgang Achnitz. Berlin, Boston 2011, Sp. 787-791.

62 Zur frühhumanistischen Übersetzungsliteratur vgl. die Ergebnisse des Marburger Repertorium (MRFH): http://www.mrfh.de; ferner Bertelsmeier-Kierst, 'Übersetzen' (vgl. Anm. 2).

$63 \mathrm{Vgl}$. Aeneas Silvius Piccolomini (Pius II) and Niklas von Wyle, The Tale of Two Lovers Eurialus and Lucretia. Ed. by E. J. Morrall. Amsterdam 1988 (Amsterdamer Publikationen zur Sprache und Literatur 77), S. 44; MRFH 42103.

64 Von den kürzeren Texten (Erstdrucke: 10-12 Bll.) wurde die Griseldis in 12 Hss. und 15 Inkunabeln (vgl. MRFH 43503), Wyles Sigismunda-Novelle in 5 Hss. und 12 Drucken (vgl. MRFH 42104) bis 1500 tradiert. 
die Müller ${ }^{65}$ und Schnyder ${ }^{66}$ noch in das Corpus der Prosaromane aufgenommen haben, der großepischen Form zurechnen kann. Einen Grenzfall stellen Novellen- und Erzählsammlungen dar, die durch eine Rahmenhandlung (z. B. Decameron) oder Vita (z. B. Aesop) eine Makrostruktur aufweisen und damit in gewisser Weise noch „romanhaft“67 genannt werden können.

Überraschend viele der im Frühhumanismus entstandenen Werke gelangen noch im 15. Jahrhundert in die Druckoffizinen, wobei sich Übersetzer wie z. B. der Ulmer Stadtarzt Heinrich Steinhöwel schon früh um eine fruchtbare Zusammenarbeit mit dem neuen Buchdruckergewerbe bemüht haben. Seine eigenen Übersetzungen wie auch Werke anderer frühhumanistischer Autoren ließ er ab 1473 bei Johann Zainer in Ulm verlegen, der in den siebziger Jahren des 15. Jahrhunderts ein literarisch anspruchsvolles und innovatives Buchsortiment in deutscher Sprache herausbrachte, zu dem neben Wyles Sigismunda und Steinhöwels Griseldis, die großen Boccaccio-Übersetzungen, Arigos Decameron und Steinhöwels Bearbeitung der claris mulieribus, sowie der Aesop gehörten.

Von diesen größeren Übersetzungen erweist sich mit Abstand Steinhöwels Aesop als der erfolgreichste Text der Inkunabelzeit. Das Werk erlebte 15 oberdeutsche Druckauflagen, von denen sich noch unmittelbar zwei weitere Bearbeitungen, der Kölner und Magdeburger Aesop ableiten, die ebenfalls zum Druck gelangten. ${ }^{68}$ Hingegen blieb den großen Boccaccio-Übertragungen im 15. Jahrhundert ein vergleichbarer Erfolg im neuen Medium des gedruckten Buches versagt, Steinhöwels Erlauchte Frauen erlebten drei Ausgaben und zwei kürzende Bearbeitungen bzw. Teilveröffentlichungen, ${ }^{69}$ das umfangreiche Decameron (Ulmer Erstdruck: 398 Bll.) nur zwei Drucke bis 1500, wurde aber im 16. Jahrhundert, als die Bücherpreise deutlich fielen, zahlreich verlegt. ${ }^{70}$ Die bei Johann Zainer gedruckten Werke Steinhöwels erzielten mit ihren qualitätsvollen

65 Jan-Dirk Müller, 'Augsburger Drucke von Prosaromanen im 15. und 16. Jahrhundert'. In: Augsburger Buchdruck und Verlagswesen. Von den Anfängen bis zur Gegenwart. Hg. v. Helmut Glier und Johannes Janota. Wiesbaden 1997, S. 337-352, hier $341 \mathrm{f}$.

66 Schnyder, 'Corpus' (Anm. 3), S. 550.

67 Gerhard Dicke, 'Steinhöwel'. In: ${ }^{2}$ Vl 9 (1995), Sp. 258-278, hier Sp. 271. [Vgl. für den ,romanhaften' Charakter des Aesop auch den Beitrag von De Bruijn in diesem Band, S 101-103).]

68 Zu Steinhöwels Aesop vgl. MRFH 43407; zum Kölner Aesop vgl. MRFH 43409; und zum Magdeburger Aesop vgl. MRFH 43410.

69 Gesamtausgaben: Ulm 1474, Augsburg 1479 und Straßburg 1488. Der Nachdruck Johann Zainers von 1476 enthält nur die Holzschnitte mit Bildbeischriften, der Mancz-Druck aus Blaubeuren nur das Kap. über Semiramis. Vgl. MRFH 43405.

70 Erstdruck: Ulm 1476-1477, dem 1490 die erste illustrierte Ausgabe Anton Sorgs folgte (vgl. MRFH 40401). 
Holzschnitten europaweite Resonanz und wurden später für französische, niederländische und spanische Ausgaben kopiert. Beliebt war auch Steinhöwels literarisches Erstlingswerk Apollonius, der in sechs Handschriften und sieben Inkunabeln tradiert wurde, ${ }^{71}$ sowie das Buch der Weisheit, das Antonius von Pforr nach der mittellateinischen Sammlung Directorium humane vitae des Johann von Capua für den württembergischen Grafen, Eberhard im Barte, übersetzte. Die Sammlung moralischer Fabeln und Geschichten wurde bereits bis 1500 in neun Handschriften und sieben Inkunabeln verbreitet. ${ }^{72}$

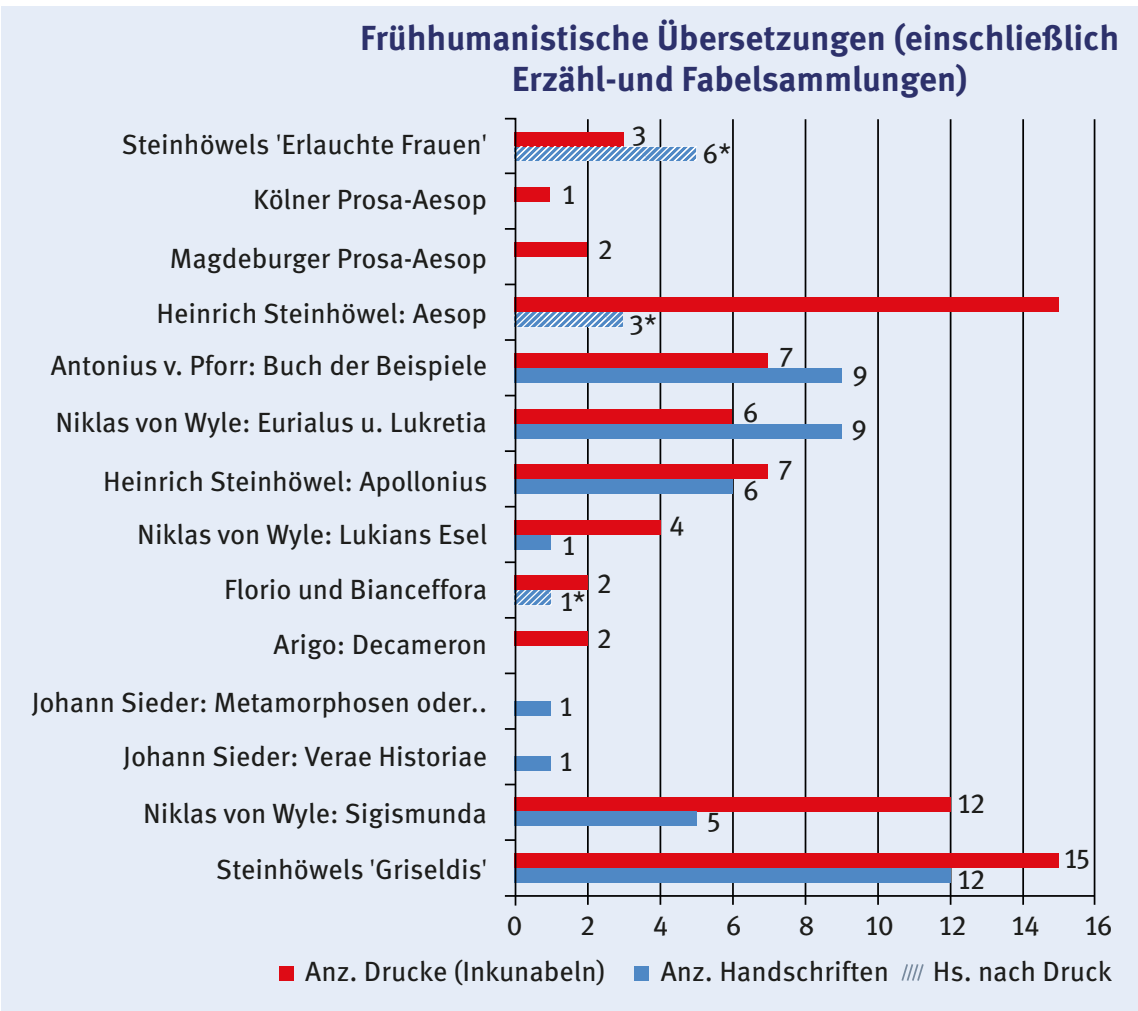

71 Tina Terrahe, Heinrich Steinhöwels Apollonius. Edition und Studien. Berlin, Boston 2013 (Frühe Neuzeit 179), S. 106-126; MRFH 43502.

72 Volker Zapf, 'Antonius von Pforr'. In: Deutsches Literatur-Lexikon 5 (vgl. Anm. 2), Sp. 1839-1844, hier 1841 f.; Ulrike Bodemann, 'Anton von Pforr, Buch der Beispiele der alten Weisen'. In: Katalog der Illustrierten Handschriften, Bd. 2, Lfg. 5, 1996, S. 360-392 (mit Abb. der Hss. und Drucke); Friedemar Geissler, 'Die Drucke des Buches der Beispiele der alten Weisen'. In: Beiträge zur Inkunabelkunde 3. F. 3 (1967), S. 18-46; http://www.handschriftencensus.de/ werke/611; GW M13190, M13178, M13180, M13181, M13184, M13187, M13192. 
Die ebenfalls für Eberhard im Barte angefertigte Übersetzung von Lukians Esel, die Niklas von Wyle nach der lateinischen Vorlage Poggio Bracciolinis verfasste, erlebte bis 1500 immerhin vier Auflagen (Erstdruck: um 1477) und eine Druckabschrift. ${ }^{73}$ Erst spät kam die anonyme Bearbeitung Florio und Bianceffora nach Boccaccios Filocolo zum Druck, die 1499 in Metz auf den Markt gebracht wurde und 1500 bereits eine zweite Auflage erlebte. Der Liebesroman avancierte im 16. Jahrhundert rasch zum ,Bestseller‘ ${ }^{74}$

Offenbar konnten, so müssen wir aus den Überlieferungszahlen schließen, vor allem diejenigen Werke rasch hohe Auflagen erzielen, die auf eine lange Stofftradition im Mittelalter (Aesop, Apollonius, Buch der Weisheit) oder bereits auf eine reiche Handschriftentradierung vor der Drucklegung (Melusine, Eurialus und Lukretia, Griseldis) zurückblicken konnten. Hingegen wurden die großen Boccaccio-Übertragungen wohl doch als $\mathrm{zu}$ neuartig empfunden, um als Unterhaltungsbuch auf dem gedruckten Buchmarkt unmittelbar Fuß zu fassen. Versagt blieb der Erfolg auch den Werken Johann Sieders, obwohl er für sehr prominente Auftraggeber, den kurfürstlichen Kanzler Johann Dalberg und Kaiser Maximilian I., zwei hellenistische Romane übersetzte, den Asinus Aureus des Apuleius und die Verae Historiae Lukians, die als frühste Repräsentanten des pikaresken und phantastisch-utopischen Romans in Deutschland gelten können. ${ }^{75}$ Erst 1538 wurde der Guldin Esel im Auftrag von Sieders Stiefbruder mit Unterstützung Kaiser Ferdinands in einer reich illustrierten Ausgabe (Augsburg: Alexander Weißenhorn, 1538) mit Holzschnitten des Hans Schäufele gedruckt, ${ }^{76}$ während die Verae Historiae nur im Widmungsexemplar Johanns von Dalberg handschriftlich überlebten.

\section{Regionale Unterschiede}

Nachdem wir uns so einen kursorischen Überblick über den Bestand an Werken der neuen Erzählprosa, ihren Stofftraditionen und ihrer Überlieferungsdichte

73 Vgl. MRFH 42115.

74 Vgl. Florio und Bianceffora. Ein gar schone hystori der hochen lieb des kuniglichen fursten Florio vnnd von seyner lieben Bianceffora. Nachdr. der Ausgabe Metz 1499. Mit einen Nachw. v. Renate Noll-Wiemann. Hildesheim, New York 1975 (Deutsche Volksbücher in Faksimiledrucken, A. 3); Silke Schünemann, Florio und Biancefforra (1499) - Studien zu einer literarischen Übersetzung, Tübingen 2005 (Frühe Neuzeit 106); MRFH 0012 (http://ww.mrfh.de/0012).

75 Vgl. Franz Josef Worstbrock, 'Sieder, Johann’. In: VL Deutscher Humanismus 2 (2013), Sp. 896-902; MRFH 0034 (http://www.mrfh.de/0034).

76 Augsburg: Alexander Weißenhorn, 1538. Abb: http://mrfh.de/abbildung.php?id=1279; Digitalisat: http://archive.org/details/apulgedichtevoneinemguldeneselsub (Göttinger Ex.). 
verschafft haben, sollen - fokussiert auf den Prosaroman - grundlegende Unterschiede zwischen der Manuskriptkultur und dem neu einsetzenden Buchdruck festgehalten werden. Zunächst überrascht die große Dichte der Handschriftenproduktion, die auf dem Gebiet des Prosaromans zu beobachten ist. Ihr Anteil übersteigt das Druckaufkommen bis 1500 noch bei weitem: 265 Handschriften stehen 125 Druckauflagen gegenüber, d. h. mehr als doppelt so oft wurden Handschriften abgeschrieben als Drucke verlegt. Vergleicht man damit das Verhältnis von Handschriften und Inkunabelauflagen, das über das MRFH für die Gesamtzahl der frühhumanistischen Übersetzungen ermittelt werden konnte, so wird der Unterschied besonders deutlich. Das hohe Handschriftenaufkommen spricht dafür, dass nicht nur die Versliteratur, sondern in großem Maße auch die neue Gattung des Prosaromans zunächst noch stark im höfischen Milieu verankert blieb und Adelsbibliotheken weiterhin eine vorrangige Rolle bei der Verbreitung der Texte im 15. Jahrhundert spielten.

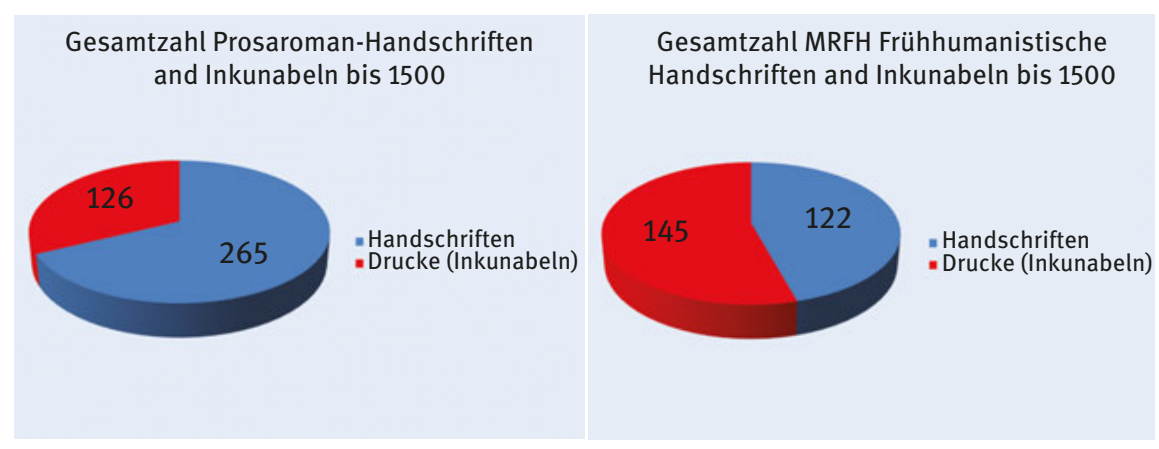

Auffällig bleibt auch die große Vielfalt an Einzelfassungen in der Handschriftentradition des 15. Jahrhunderts. So haben sich beispielsweise zum Trojabuch zwölf Fassungen in der Manuskriptkultur erhalten, während sich auf dem gedruckten Buchmarkt oftmals nur eine einzige Fassung durchsetzt, oder es gibt sprachlich klar abgegrenzte Räume, für die wie z. B. beim Buch von Troja je eine oberdeutsche und niederdeutsche Druckfassung auf den Markt kommt. Meistens geht jedoch den niederdeutschen Auflagen eine oberdeutsche Drucktradition voraus. So basieren die niederdeutschen Inkunabeln u.a. von Hartliebs Alexander (Erstausgabe 1472) 1477-78, Die sieben weisen Meister (Erstdruck 1473) oder Thürings Melusine (Erstdruck 1474) 1478 auf oberdeutschen Ausgaben. Nur wenn die Vorlage dem niederländisch-burgundischen Raum entlehnt wurde, wie z. B. bei der niederdeutschen Druckausgabe von Paris und Vienna, die Gerhard Leeu 1488 in Antwerpen druckte, geht der niederdeutsche Raum voran bzw. etabliert sich eine singuläre Drucktradition im Norden. 
Im oberdeutschen Raum lässt sich vor 1500 noch eine deutliche Zweiteilung in Druckorte mit durchgeführter neuhochdeutscher Diphthongierung (Zentren: Augsburg, Nürnberg) und den Druckorten mit Beibehaltung der alten Längen (Zentren: Straßburg, Ulm, Basel) beobachten. ${ }^{77}$ Beide Räume verfügten alsbald über eigene Vertriebsnetze, bei denen von Straßburg, Ulm oder Basel aus der alemannisch-schwäbische Raum, einschließlich der Schweiz, sowie die mittelrheinischen Gebiete beliefert wurden, während Drucker aus Augsburg und Nürnberg den ostschwäbischen, bairisch-österreichischen sowie den fränkisch-ostmitteldeutschen Raum mit Büchern versorgten. ${ }^{78}$

Bemerkenswert ist aber nicht nur die sprachlich-geographische Zäsur, sondern die deutschsprachigen Drucke aus dem Südwesten unterscheiden sich auch im Layout deutlich von den Augsburger Inkunabeln. Augsburg war führend in der deutschsprachigen Buchproduktion und spezialisierte sich, abgesehen von Bibeln und dem breiten Strom religiöser Gebrauchsliteratur, auf unterhaltende Prosa, deren Markenzeichen zunehmend kleinformatige Holzschnitte wurden, wobei man bei weniger umfangreichen Werken bereits in der Inkunabelzeit zum Quartformat überging. ${ }^{79}$ Während die Augsburger Drucke auf dem Sektor der unterhaltenden Prosa somit alsbald ein eigenes Profil entwickelten, blieben die Drucke aus den südwestdeutschen Metropolen (Basel, Ulm und Straßburg) in ihrem Layout viel stärker der Handschriftenkultur verhaftet, die sich gerade am Oberrhein, in der Schweiz und in Schwaben bevorzugt an französischen und burgundisch-flämischen Vorbildern orientierte.

Nicht selten scheinen den dort ansässigen Inkunabeldruckern Handschriften aus Adelsbibliotheken vorgelegen $\mathrm{zu}$ haben, aus denen sie aufwendige Elemente wie heraldischen Buchschmuck, Rankenbordüren und die Bildvorlagen für ihre Holzschnitte entlehnten. So benutzte Conrad Fyner für die Uracher Erstausgabe (um 1481) vom Buch der Weisheit, die er mit 128 Holzschnitten aufwendig

77 Im 16. Jahrhundert ändert sich dies, weil auch alemannische und westdeutsche Drucker, vor allem unter dem Konkurrenzdruck der ostmd. Bibelausgaben, schreibsprachlich die Diphthongierung durchführen.

78 Gut belegt z. B. bei der Drucküberlieferung der Melusine oder beim Decameron. Vgl. Christa Bertelsmeier-Kierst, Griseldis in Deutschland. Studien zu Steinhöwel und Arigo. Heidelberg 1988 (GRM-Beiheft 8), S. 68.

79 Vgl. Müller (vgl. Anm. 65); Hans-Jörg Künast, Getruckt zu Augspurg: Buchdruck und Buchhandel zwischen 1468 und 1555. Tübingen 1997 (Studiana Augustana 8). [Siehe für die Benutzung des Quartformats in England den Beitrag von Boffey (Seite 138), für die Verwendung des Folioformats bei französischen Artusromanen den Beitrag von Montorsi (Seite 174) und für das Oktavformat in Dänemark den Beitrag von Richter (Seite 330) in diesem Band.] 
illustrieren ließ,,$^{80}$ als Vorlage Miniaturen aus der Heidelberger Handschrift (Cpg 466), die aus der unmittelbaren Umgebung des württembergischen Hofes, vielleicht aus dem Besitz von Eberhards Schwägerin, Margarethe von Savoyen, stammt. ${ }^{81}$ Noch anspruchsvoller gestaltete 1483 Lienhart Holl die Ulmer Erstausgabe vom Buch der Weisheit, deren Holzschnitte Lilli Fischel „als Meisterwerke

\section{Antonius von Pforr : Buch der Beispiele der alten Weisen}

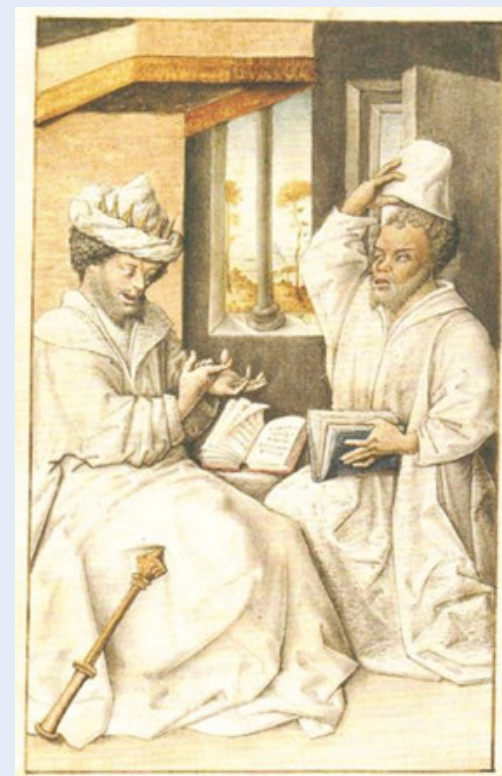

Heidelberg, UB, Cpg 84, 1474/75 aus dem Besitz Eberhards von Württemberg (im Barte)

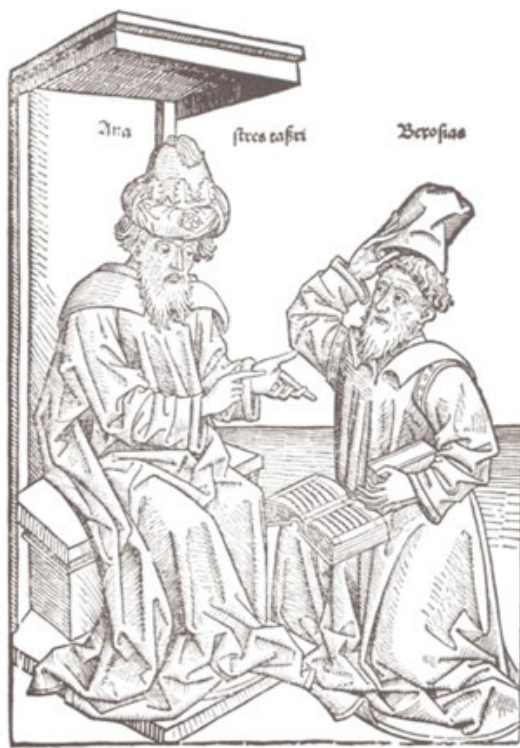

Ulm, Lienhart Holl, 1483

Holzschnitte nach dem Manuskript Eberhards im Barte

80 Peter Amelung, Der Frühdruck im deutschen Südwesten. 1473-1500. Eine Ausstellung der Württembergischen Landesbibliothek Stuttgart. Bd. I: Ulm. Stuttgart 1979, Kat. Nr. 141, Abb. 218, 221 und 223.

81 Vgl. Mathias Miller und Karin Zimmermann, Die Codices Palatini germanici in der Universitätsbibliothek Heidelberg (Cod. Pal. germ. 304-495). Wiesbaden 2007, S. 500 f.; Effinger und Losert (vgl. Anm. 25), S. 58-60 und Abb. 30; Bodeman (vgl. Anm. 72): Digitalisat: http://digi. ub.uni-heidelberg.de/diglit/cpg466. 
deutscher Graphik und des ulmischen Stils“ ${ }^{\circ 2}$ bezeichnete. ${ }^{83}$ Holl ersetzte die halbseitigen Illustrationen der Fyner-Ausgaben durch ganzseitige Holzschnitte, deren Vorlagen unmittelbar dem Heidelberger Codex (Cpg 84) entnommen wurden, dessen aufwendige, im burgundisch-niederländischen Stil angefertigte Miniaturen weitgehend dem für Eberhard von Württemberg etwas früher angefertigten Pergamentcodex (Chantilly, Musée Condé, Ms. 460) entsprechen und entweder für Graf Eberhard selbst oder seine Mutter, die Erzherzogin Mechthild von der Pfalz, verfasst wurde, in deren Besitz sich der Cpg 84 offenbar später befand. ${ }^{84}$ Ein kleiner Teil der Auflage wurde darüber hinaus in Holls Erstausgabe auf Pergament gedruckt. ${ }^{85}$ Auch für die Ulmer Ausgabe von Heinrich Steinhöwels Erlauchten Frauen (1473/74), deren künstlerisch hochwertige Holzschnitte ,auf französisch-burgundische Vorbilder zurückgehen“, ${ }^{86}$ wurde die Widmungsvorrede an Eleonore von Schottland, die Gemahlin Sigmunds von Tirol, in einigen Exemplaren auf Pergament gedruckt.

Im Südwesten behielt man darüber hinaus noch Normalfolio als Standarddruckformat bei, das ebenfalls in der Handschriften-Produktion die Regel war. ${ }^{87}$ Bei besonders repräsentativen Drucken konnte aber auch für Werke der erzählenden Prosa das sog. ,Königsformat‘ verwendet werden, wie der Basler Erstdruck vom Ritter vom Turn im Auftrag Johann Bergmanns von Olpe mit seinem beeindruckenden Format $(42$ × $29 \mathrm{~cm})$ demonstriert. ${ }^{88}$ Dieses Format war zuvor ein Markenzeichen der Bilderhandschriften Diepold Laubers in Hagenau gewesen, der vor allem für Konrads Trojanerkrieg und seine Historienbibeln dieses Überformat wählte. Das für Maximilian I. geschriebene Ambraser Heldenbuch, eine Sammlung mittelhochdeutscher Versepik, wird im frühen 16. Jahrhundert nochmals an diese Tradition anknüpfen.

Wie diese Beispiele erhellen, orientierte sich die anspruchsvolle Inkunabelproduktion im Südwesten noch deutlich am Handschriftenpublikum, dessen Geschmack nachhaltig durch die Manuskriptkultur Frankreichs und Burgunds im

82 Lilli Fischel, Bilderfolgen des frühen Buchdruck. Studien zur Inkunabel-Illustration in Ulm und Straßburg. Konstanz, Stuttgart 1963, S. 63-91, hier 91.

$83 \mathrm{Zu}$ den Ulmer Ausgaben Holls: Amelung (vgl. Anm. 80), Nr. 141 (m. Abb. 216, 217 und 219), Nr. 142 (m. Abb. 220, 222, 224 und 225).

84 Vgl. Zimmermann (vgl. Anm. 25), S. 213 f.; Effinger und Losert (Anm. 25), S. 32 f., 36 f. und Abb. 2, 12 f.; Bodemann (vgl. Anm. 72); Digitalisat: http://digi.ub.uni-heidelberg.de/diglit/ cpg84.

85 Amelung (vgl. Anm. 80), S. 297.

86 Amelung (vgl. Anm. 80), S. 76.

87 Backes (vgl. Anm. 39), S. 118.

88 Vgl. Schmitt (vgl. Anm. 45) und Peter Jörg Becker, 'Diepold Lauber'. In: Aderlass und Seelentrost (vgl. Anm. 23), S. 130-135, ins. Nr. 62. 
15. Jahrhundert geprägt wurde. Die aufwendig gestalteten Inkunabeln aus Basel, Ulm oder Straßburg dürften somit um ein Vielfaches teurer als vergleichbare Druckexemplare aus Augsburg gewesen sein und sprachen ausschließlich die vermögendsten Käuferschichten aus dem Adel und den Eliten der Metropolen an. Unter den ,Massen“ war diese Literaturproduktion also auch in der Inkunabelzeit noch lange nicht ,zuhause“ und dies zeigt einmal mehr, wie irreführend der Begriff des ,Volksbuchs“ ist, wenn er bereits auf die Inkunabelzeit angewendet wird, wo das gedruckte Buch noch mit dem handschriftlichen konkurrierte und man vor allem im Südwesten durch kostbaren Buchschmuck Elemente der Manuskriptkultur im Wiegendruck konservierte. Erst im Laufe des 16. Jahrhunderts wird sich dann speziell auf dem Gebiet der unterhaltenden Prosa das Buch als Massenerzeugnis auch für breitere städtische Schichten durchsetzen.

\section{Fazit}

Noch wenig wurde für den aufkommenden Buchmarkt die Frage untersucht, wie sich der mediale Wandel auf die Texte und die sie begleitenden Paratexte auswirkte. Es fällt auf, dass gerade Augsburger Drucke schon relativ früh Bearbeitungstendenzen zeigen, wie sie dann im Bereich der Erzählprosa im Laufe des 16. Jahrhunderts in großem Stil für gedruckte Erzeugnisse üblich werden. So wurde in Steinhöwels Apollonius nach dem Erstdruck 1471 bei Günter Zainer der Reimprolog mit dem kunstvollen Akrostichon bereits im nachfolgenden Druck Johann Bämlers und allen weiteren Ausgaben unterdrückt, weil man ihn offenbar als „veraltet oder unpassend für das intendierte Publikum früher deutscher Druckprosa“ empfand. ${ }^{89}$ Nur in der vom Autor überwachten und vermutlich mitfinanzierten Ulmer Erstausgabe wird Steinhöwels Aesop zweisprachig gesetzt, während die nachfolgenden Augsburger Drucker, offenbar aus marktrelevanten Gründen, den Text nur noch einsprachig, auf Latein oder Deutsch, verlegten. Anton Sorg versah 1490 seine Augsburger Ausgabe des Decameron nicht nur mit absatzfördernden Holzschnitten, sondern entfernte stillschweigend auch die Novelle VII,10, um vermutlich der Kritik kirchlicher Kreise zuvorzukommen. Er leitete damit einen Prozess ein, bei dem vor allem in Ausgaben des 16. Jahrhunderts der einheitliche Kunstcharakter des Decameron mehr und mehr zerstört wurde, indem die Drucker Passagen nach Gutdünken strichen oder fremde Geschichten hinzufügten und ab der Ausgabe von 1535 die Rahmenhandlung fast vollständig unterdrückten, so dass das Werk sich in den Druckausgaben des 16. Jahrhunderts den

89 Terrahe, Apollonius (Anm. 71), S. 142. 
herkömmlichen Schwanksammlungen annäherte. ${ }^{90}$ Auch für das Buch der Weisheit oder den Ritter vom Turn, die beide im Südwesten beeindruckende Prachtausgaben bis 1500 erlebten, lassen sich ähnliche Eingriffe beobachten und es wäre eine lohnende Aufgabe, diese Veränderungen literarischer Texte im frühen Buchdruck des 15. und 16. Jahrhunderts systematisch zu untersuchen. Die oft angemerkten ,Trivialisierungstendenzen“ im frühneuhochdeutschen Prosaroman zeigen sich m.E. verstärkt in den direkt für den Buchmarkt hergestellten Texten, etwa in den anonym in Augsburg verlegten Prosafassungen von Tristrant und Isalde und Herr Wigoleis vom Rade. Ebenso lässt sich beobachten, dass in den Druckauflagen nicht selten Werke anonym erscheinen, denen in der handschriftlichen Tradierung noch Angaben zum Autor oder Widmungsempfänger in Vorreden vorausgingen. Um diese, teils gravierenden Veränderungen durch den frühen Buchdruck sichtbar zu machen, bedarf es einer umfassenden Untersuchung, die nicht nur die Frühdrucke, sondern auch die handschriftliche Tradierung der Texte eingehend berücksichtigen müsste. Erst auf diesem Fundament können die mediengeschichtlichen Veränderungen im Druckzeitalter eingehend dokumentiert und die Auswirkungen auf die deutschsprachige Literatur in Gänze erfasst werden.

\section{Bibliografie}

Achnitz, Wolfgang, 'Das Feld der literarischen Kleinformen im Mittelalter'. In: Deutsches Literatur-Lexikon: Das Mittelalter, Bd. 5: Epik. Hg v. Wolfgang Achnitz. Berlin, Boston 2013, S. XXVII-XXXVIII.

Aeneas Silvius Piccolomini (Pius II) and Niklas von Wyle, The Tale of Two Lovers Eurialus and Lucretia. Ed. by E.J. Morrall. Amsterdam 1988 (Amsterdamer Publikationen zur Sprache und Literatur 77).

Andersen, Peter H., 'Wigbolds Alexanderbuch - Eine Pioniertat'. In: Eulenspiegel trifft Melusine. Der frühneuhochdeutsche Prosaroman im Licht neuer Forschungen und Methoden. Hg. v. Catherine Drittenbass und André Schnyder. Amsterdam, New York 2010 (Chloe 42), S. 183-200.

Amelung, Peter, Der Frühdruck im deutschen Südwesten. 1473-1500. Eine Ausstellung der Württembergischen Landesbibliothek Stuttgart. Bd. I. Ulm. Stuttgart 1979.

Alfen, Clemens, Petra Flochler und Elisabeth Lienert, 'Deutsche Trojatexte des 12.-16. Jhs., Repertorium'. In: Die deutsche Trojaliteratur des Mittelalters und der frühen Neuzeit. Materialien und Untersuchungen. Hg. v. Horst Brunner. Wiesbaden 1990 (Wissensliteratur im Mittelalter 3), S. 104-111.

Altenhöfer, Florian, ‘Tristan'. In: Deutsches Literatur-Lexikon: Das Mittelalter, Bd. 5: Epik. Hg. v. Wolfgang Achnitz. Berlin, Boston 2013, Sp. 1966.

90 Vgl. Bertelsmeier-Kierst, Griseldis (Anm. 78), S. 174 f. 
Backes, Martina, Das literarische Leben am kurpfälzischen Hof zu Heidelberg im 15. Jahrhundert. Ein Beitrag zur Gönnerforschung des Spätmittelalters. Tübingen 1992.

Backes, Martina, Fremde Historien. Untersuchungen zur Überlieferungs- und Rezeptionsgeschichte französischer Erzählstoffe im deutschen Mittelalter. Tübingen 2004 (Hermaea 103).

Bastert, Bernd (Hg.), Herzog Herpin: Kritische Edition eines spätmittelalterlichen Prosaepos. Berlin 2014 (TMA 51).

Becker, Peter Jörg, 'Diepold Lauber'. In: Aderlass und Seelentrost. Die Überlieferung deutscher Texte im Spiegel Berliner Handschriften und Inkunabeln. Hg. v. Peter Jörg Becker und Eef Overgaauw. Mainz 2003, S. 130-135.

Becker, Peter Jörg, 'Elisabeth von Nassau-Saarbrücken: Herzog Herpin'. In: Aderlass und Seelentrost. Die Überlieferung deutscher Texte im Spiegel Berliner Handschriften und Inkunabeln. Hg. v. Peter Jörg Becker und Eef Overgaauw. Mainz 2003, S. 135-137.

Beckers, Hartmut, 'Reinolt von Montalban'. In: ${ }^{2}$ VL 7 (1989), Sp. 1208-1214.

Benzinger, Rudolf et al. (Hgg.), Der gute Gerhard Rudolf von Ems in einer anonymen Prosaauflösung und die lateinische und deutsche Fassung der Gerold-Legende Albrechts von Bonstetten. Nach den Handschriften Reg. 0157 und Reg. O 29a und b im Thüringischen Hauptstaatsarchiv Weimar. Berlin 2001 (DTM 81).

Bertelsmeier-Kierst, Christa, Griseldis in Deutschland. Studien zu Steinhöwel und Arigo. Heidelberg 1988 (GRM-Beiheft 8).

Bertelsmeier-Kierst, Christa, 'Zur Rezeption des lateinischen und volkssprachlichen Boccaccio im deutschen Frühhumanismus'. In: Giovanni Boccaccio in Europa. Studien zu seiner Rezeption in Spätmittelalter und Früher Neuzeit. Hg. v. Achim Aurnhammer und Rainer Stillers. Wiesbaden 2014 (Wolfenbütteler Abh. zur Renaissanceforschung 31), S. 131-154.

Bertelsmeier-Kierst, Christa, 'Erzählen in Prosa. Zur Entwicklung des deutschen Prosaromans bis 1500'. In: ZfdA 143 (2014), S. 141-165.

Bertelsmeier-Kierst, Christa, 'Übersetzen im deutschen Frühhumanismus. Ergebnisse des MRFH zur Einbürgerung humanistischer und antiker Autoren bis 1500'. In: Humanistische Antikenübersetzung und frühneuzeitliche Poetik in Deutschland (1450-1620). Hg. v. Regina Töpfer, Johannes Klaus Kipf und Jörg Robert. Berlin, Boston 2017 (Frühe Neuzeit 211), S. 125-150.

Bertelsmeier-Kierst, Christa, 'Rekontextualisierung des Wissens. Res fictae und res factae im vormodernen Roman am Beispiel von Thürings Melusine’. In: Enzyklopädisches Erzählen und vormoderne Romanpoetik. Hg. v. Mathias Herweg, Johannes Klaus Kipf und Dirk Werle (Wolfenbütteler Forschungen) [im Druck].

Bloh, Ute von, Ausgerenkte Ordnung. Vier Prosaepen aus dem Umkreis der Gräfin Elisabeth von Nassau-Saarbrücken: Herzog Herpin, Loher und Maller, Huge Scheppel, Königin Sibille. Tübingen 2002 (MTU 119).

Bloh, Ute von, Kurt Gärtner und Michael Heintze, 'Lohier et Malart - Loher und Maller: Vorschläge zu einer Edition des Epos'. In: Zwischen Deutschland und Frankreich. Elisabeth von Lothringen, Gräfin von Nassau-Saarbrücken. Hg. v. Wolfgang Haubrichs und Hans-Walter Herrman, unter Mitw. v. Gerhard Sauder. St. Ingbert 2002, S. 427-458.

Bloh, Ute von (Hg.), Loher und Maller: Kritische Edition eines spätmittelalterlichen Prosaepos. Berlin 2013 (TMA 50).

Bloh, Ute von und Bernd Bastert, Loher und Maller - Herzog Herpin: Kommentar und Erschließung. Berlin 2017 (TMA 55). 
Bodemann, Ulrike, 'Anton von Pforr, Buch der Beispiele der alten Weisen'. In: Katalog der Illustrierten Handschriften, Bd. 2, Lfg. 5, 1996, S. 360-392.

Cramer, Thomas, 'Aspekte des höfischen Romans im 14. Jahrhundert'. In: Zur deutschen Literatur und Sprache des 14. Jahrhunderts. Dubliner Colloquium 1981. Hg. v. Walter Haug, Timothy R. Jackson und Johannes Janota. Heidelberg 1983, S. 208-220.

Deifuß, Holger, Hystoria von dem wirdigen ritter sant Wilhelm. Kritische Edition und Untersuchung einer frühneuhochdeutschen Prosauflösung. Frankfurt 2005 (Germ. Arbeiten zu Sprache und Kulturgesch. 45).

Dicke, Gerhard, 'Steinhöwel'. In: ${ }^{2}$ VI 9 (1995), Sp. 258-278.

Dietl, Cora, 'Wilhelm von Österreich'. In: ${ }^{2}$ VL 10 (1999), Sp. 1114-1116.

Effinger, Maria und Kerstin Losert (Hg.), Mit schönen figuren. Buchkunst im deutschen Südwesten. Heidelberg 2014.

Ertzdorff, Xenia von, Romane und Novellen des 15. und 16. Jahrhunderts in Deutschland. Darmstadt 1989.

Ertzdorff, Xenia von, 'Pontus und Sidonia'. In: ${ }^{2}$ VL 7 (1989), Sp. 780-782.

Faks. Bayerische Staatsbibliothek, Cgm 9220. Mit einer Einf. v. Klaus Grubmüller und einer Beschr. v. Ulrich Montag. München 1999 (Patrimonia 154).

Fischel, Lilli, Bilderfolgen des frühen Buchdruck. Studien zur Inkunabel-Illustration in Ulm und Straßburg. Konstanz, Stuttgart 1963.

Florio und Bianceffora. Ein gar schone hystori der hochen lieb des kuniglichen fursten Florio vnnd von seyner lieben Bianceffora. Nachdr. der Ausgabe Metz 1499. Mit einen Nachw. v. Renate Noll-Wiemann. Hildesheim, New York 1975 (Deutsche Volksbücher in Faksimiledrucken, A. 3).

Gantert, Klaus, 'Der Heiligen Leben'. In: Aderlass und Seelentrost. Die Überlieferung deutscher Texte im Spiegel Berliner Handschriften und Inkunabeln. Hg. v. Peter Jörg Becker und Eef Overgaauw. Mainz 2003, S. 218-221.

Geissler, Friedemar, 'Die Drucke des Buches der Beispiele der alten Weisen'. In: Beiträge zur Inkunabelkunde 3. F. 3 (1967), S. 18-46.

Geith, Karl-Ernst, 'Zürcher Buch vom heiligen Karl’. In: ${ }^{2}$ VL 10 (1999), Sp. 1597-1600 und 11 (2004), Sp. 1697.

Gerhardt, Christoph, 'Willehalm (Prosaroman)’. In: ${ }^{2}$ VL 10 (1999), Sp. 1151-1154.

Gerhardt, Christoph, Rez. zu: Holger Deifuß, Hystoria von dem wirdigen ritter sant Wilhelm. In: ZfdA 136 (2007), S. 263-271.

Grubmüller, Klaus, 'Hartlieb, Johannes'. In: ${ }^{2}$ VL 3 (1981), Sp. 480-496 und 11 (2004), Sp. 589-590.

Hahn, Reinhard, 'Pontus und Sidonia in der Berner Fassung'. In: Daphnis 32 (2003), S. 289-350.

Haubrichs, Wolfgang und Hans-Walter Herrmann, unter Mitw. v. Gerhard Sauder (Hg.), Zwischen Deutschland und Frankreich. Elisabeth von Lothringen, Gräfin von NassauSaarbrücken. St. Ingbert 2002.

Haubrichs, Wolfgang, 'Kurze Forschungsgeschichte zum literarischen Werk Elisabeths'. In: Zwischen Deutschland und Frankreich. Elisabeth von Lothringen, Gräfin von NassauSaarbrücken. Hg. v. Wolfgang Haubrichs und Hans-Walter Herrman, unter Mitw. v. Gerhard Sauder. St. Ingbert 2002, S. 17-48.

Haubrichs, Wolfgang, 'Die vier Prosahistorien Elisabeths - Skizzierung ihres Inhalts'. In: Zwischen Deutschland und Frankreich. Elisabeth von Lothringen, Gräfin von Nassau- 
Saarbrücken. Hg. v. Wolfgang Haubrichs und Hans-Walter Herrman, unter Mitw.

v. Gerhard Sauder. St. Ingbert 2002, S. 11-16.

Haubrichs, Wolfgang, 'Mahl und Krieg. Die Erzählung der Adelskultur in den Texten und

Bildern des Hamburger Huge Scheppel der Elisabeth von Lothringen, Gräfin von Nassau-

Saarbrücken'. In: Eulenspiegel trifft Melusine. Der frühneuhochdeutsche Prosaroman im

Licht neuer Forschungen und Methoden. Hg. v. Catherine Drittenbass und André

Schnyder. Amsterdam, New York 2010 (Chloe 42), S. 201-216.

Heinzle, Joachim, Wandlungen und Neuansätze im 13. Jahrhundert. Geschichte der deutschen Literatur von den Anfängen bis zum Beginn der Neuzeit. Bd. 2, T. 2. Tübingen ${ }^{2} 1994$.

Horváth, Eva und Hans-Walter Storck (Hg.), Von Rittern, Bürgern und von Gottes Wort.

Hamburg 2002 (Schriften aus dem Antiquariat Günther, Hamburg, 2).

Jahn, Bruno, 'Elisabeth'. In: Deutsches Literatur-Lexikon: Das Mittelalter, Bd. 5: Epik. Hg. v. Wolfgang Achnitz. Berlin, Boston 2013, Sp. 1494-1505.

Janota, Johannes, Orientierung durch volkssprachliche Schriftlichkeit (1280/

90-1380/90). Geschichte der deutschen Literatur von den Anfängen bis zum Beginn der Neuzeit. Hg. v. Joachim Heinzle. Band III.1 Tübingen 2004.

Kocher, Ursula, Boccaccio und die deutsche Novellistik. Formen der Transposition italienischer "novelle" im 15. u. 16. Jahrhundert. Amsterdam 2005.

Kornrumpf, Gisela, 'Meister Wichwolt'. In: Killy ${ }^{2} 12$ (2011), S. 367-368.

Kreutzer, Hans-Joachim, 'Marquart von Stein'. In: ${ }^{2}$ VL 6 (1987), Sp. 129-135.

Künast, Hans-Jörg, Getruckt zu zu Augspurg: Buchdruck und Buchhandel zwischen 1468 und 1555. Tübingen 1997 (Studiana Augustana 8).

Künast, Hans-Jörg und Ursula Rautenberg, 'Typographie und Leseweisen. Überlegungen zu den Melusine-Ausgaben der Frankfurter Druckoffizinen[. . .]'. In: Eulenspiegel trifft Melusine. Der frühneuhochdeutsche Prosaroman im Licht neuer Forschungen und Methoden. Hg. v. Catherine Drittenbass und André Schnyder. Amsterdam, New York 2010 (Chloe 42), S. 341-359.

Malm, Mike, 'Mair von Nördlingen, Hans'. In: Deutsches Literatur-Lexikon. Das Mittelalter, Bd. 3: Reiseberichte und Geschichtsschreibung. Hg. v. Wolfgang Achnitz. Berlin, Boston 2011, Sp. 475-477.

Malm, Mike, 'Fuetrer’. In: Deutsches Literatur-Lexikon: Das Mittelalter, Bd. 5: Epik. Hg. v. Wolfgang Achnitz. Berlin, Boston 2013, Sp. 1867-1873.

Malm, Mike 'Paris und Vienna'. In: Deutsches Literatur-Lexikon: Das Mittelalter, Bd. 5: Epik. Hg. v. Wolfgang Achnitz. Berlin, Boston 2013, Sp. 1901-1904.

Malm, Mike, 'Marquart von Stein'. In: Deutsches Literatur-Lexikon: Das Mittelalter, Bd. 5: Epik. Hg. v. Wolfgang Achnitz. Berlin, Boston 2013, Sp. 1925-1929.

Malm, Mike, ‘Thüring von Ringoltingen'. In: Deutsches Literatur-Lexikon: Das Mittelalter, Bd. 5: Epik. Hg. v. Wolfgang Achnitz. Berlin, Boston 2013, Sp. 1598-1604.

Meier, Jürgen, 'Paris und Vienna'. In: ${ }^{2}$ VL 7 (1989), Sp. 306-309.

Miller, Mathias und Karin Zimmermann, Die Codices Palatini germanici in der Universitätsbibliothek Heidelberg (Cod. Pal. germ. 304-495). Wiesbaden 2007.

Mühlethaler Jean-Claude und André Schnyder (Hg.), 550 Jahre dt. Melusine. Couldrette und Thüring von Ringoltingen. Bern 2008 (Beitr. der wiss. Tagung der Universitäten Bern und Lausanne).

Müller, Jan-Dirk, 'Volksbuch/Prosaroman im 15./16. Jahrhundert - Perspektiven der Forschung.' In: Internationales Archiv für Sozialgeschichte der Literatur: Sonderheft Forschungsreferate 1 (1985), S. 1-128. 
Müller, Jan-Dirk, ‘Thüring von Ringoltingen’. In: ${ }^{2}$ VL 9 (1995), Sp. 908-914.

Müller, Jan-Dirk, ‘Augsburger Drucke von Prosaromanen im 15. und 16. Jahrhundert'. In: Augsburger Buchdruck und Verlagswesen. Von den Anfängen bis zur Gegenwart. Hg. v. Helmut Glier und Johannes Janota. Wiesbaden 1997, S. 337-352.

Müller, Jan-Dirk, ‘Prosaroman'. In: Reallexikon der deutschen Literaturwissenschaft 3 (2003), S. 174-177.

Müller, Mario, 'Pontus und Sidonia'. In: Deutsches Literatur-Lexikon: Das Mittelalter, Bd. 5: Epik. Hg. v. Wolfgang Achnitz. Berlin, Boston 2013, Sp. 1575-1598.

Nyholm, Kurt, ‘Füetrer: Ulrich'. In: ${ }^{2}$ VL 2 (1980), Sp. 999-1007.

Palmer, Nigel, Visio Tnugdali. The German and Dutch Translations and Their Circulation in the Later Middle Ages. München 1982 (MTU 76).

Palmer, Nigel, ‘Tundalus'. In: ${ }^{2}$ VL 9 (1995), Sp. 1142-1146.

Philipowski, Katharina, 'Reinolt von Montelban'. In: Deutsches Literatur-Lexikon: Das Mittelalter, Bd. 5: Epik. Hg. v. Wolfgang Achnitz. Berlin, Boston 2013, Sp. 1605-1608.

Plate, Bernward, Gregorius auf dem Stein, Frühneuhochdeutsche Prosa (15. Jh.) nach dem mittelhochdeutschen Versepos Hartmanns von Aue. Darmstadt 1983 (Texte zur Forschung 39).

Reinhold, Konrad, 'Willehalm'. In: Deutsches Literatur-Lexikon: Das Mittelalter, Bd. 5: Epik. Hg. v. Wolfgang Achnitz. Berlin, Boston 2013, Sp. 17283-1784.

Röcke, Werner, 'Hartlieb, Johannes'. In: Killy² 12 (2011), S. 35-36.

Rosenfeld, Hans-Friedrich, 'Alexius [Nachtrag] '. In: ${ }^{2}$ VL 11 (2004), Sp. 61-62.

Rubini Messerli, Luisa, Boccaccio deutsch. Die Dekameron-Rezeption in der deutschen Literatur (15.-17. Jahrhundert). Amsterdam 2012 (Chloe 45).

Sauder, Gerhard, 'Die Rezeption der Prosaromane Elisabeths von Nassau-Saarbrücken - Vom "Volksbuch" bis zur Romantik'. In: Zwischen Deutschland und Frankreich. Elisabeth von Lothringen, Gräfin von Nassau-Saarbrücken. Hg. v. Wolfgang Haubrichs und Hans-Walter Herrmann, unter Mitw. v. Gerhard Sauder. St. Ingbert 2002, S. 569-589.

Schmitt, Anneliese, 'La Tour-Landry, Geoffrey de: Der Ritter vom Turm, deutsche Bearbeitung von Marquard Stein'. In: Aderlass und Seelentrost. Die Überlieferung deutscher Texte im Spiegel Berliner Handschriften und Inkunabeln. Hg. v. Peter Jörg Becker und Eef Overgaauw. Mainz 2003, S. 143-144.

Schmitz, Markus, Die legent vnd dz leben des hochgelobten manlichen ritters sant joergen. Kritische Neuedition und Interpretation einer alemannischen Prosalegende des heiligen Georg im 15. Jh.). Berlin 2013 (TMA 49).

Schneider, Karin, 'Buch von Troja nach Guido de Columnis'. In: ${ }^{2}$ VL I (1978), Sp. 1101-1104.

Schneider, Karin, 'Buch von Troja I'. In: ${ }^{2}$ VL 1 (1978), Sp. 1100 und $^{2}$ VL 11 (2004), Sp. 300.

Schnyder, André, 'Der deutsche Prosaroman des 15. und 16. Jahrhunderts'. In: Eulenspiegel trifft Melusine. Der frühneuhochdeutsche Prosaroman im Licht neuer Forschungen und Methoden. Hg. v. Catherine Drittenbass und André Schnyder. Amsterdam, New York 2010 (Chloe 42), S. 11-39.

Schnyder, André, 'Das Corpus der frühneuhochdeutschen Prosaromane: Eine tabellarische Übersicht als Problemaufriss'. In: Eulenspiegel trifft Melusine. Der frühneuhochdeutsche Prosaroman im Licht neuer Forschungen und Methoden. Hg. v. Catherine Drittenbass und André Schnyder. Amsterdam, New York 2010 (Chloe 42), S. 545-556.

Schünemann, Silke, Florio und Biancefforra (1499) - Studien zu einer literarischen Übersetzung. Tübingen 2005 (Frühe Neuzeit 106).

Steinhoff, Hans-Hugo, ‘Elisabeth von Nassau-Saarbrücken'. In: ${ }^{2}$ VL 2 (1980), Sp. 482-488. 
Steinhoff, Hans-Hugo, 'Magelone'. In: ${ }^{2}$ VL 5 (1985), Sp. 1142-1148.

Steinhoff, Hans-Hugo, 'Mair, Hans von Nördlingen'. In: ${ }^{2}$ VL 5 (1985), Sp. 1180-1183.

Steinhoff, Hans-Hugo, 'Tristan'. In: ${ }^{2}$ VL 9 (1995), Sp. 1060-11061.

Streun, Kristina, 'Pontus und Sidonia (C)'. In: ${ }^{2}$ VL 11 (2004), Sp. 1259-1260.

Stöllinger-Löser, Christine, 'Barlaam und Josaphat’. In: ${ }^{2}$ VL 11 (2004), Sp. 215-219.

Storck, Hans-Walther, 'Die handschriftliche Überlieferung der Werke Elisabeths von Nassau-

Saarbrücken und die malerische Ausstattung der Handschriften'. In: Zwischen

Deutschland und Frankreich. Elisabeth von Lothringen, Gräfin von Nassau-Saarbrücken.

Hg. v. Wolfgang Haubrichs und Hans-Walter Herrmann, unter Mitw. v. Gerhard Sauder. St. Ingbert 2002, S. 591-606.

Stridde, Christine, 'Tundalus'. In: Deutsches Literatur-Lexikon. Das Mittelalter, Bd. 2: Das geistliche Schrifttum des Spätmittelalters. Hg. v. Wolfgang Achnitz. Berlin, Boston 2011, Sp. 787-791.

Stridde, Christine, 'Zürcher Buch vom heiligen Karl'. In: Deutsches Literatur-Lexikon: Das Mittelalter, Bd. 5: Epik. Hg. v. Wolfgang Achnitz. Berlin, Boston 2013, Sp. 1781-1783.

Terrahe, Tina, 'Eine neue Handschrift der Melusine Thürings von Ringoltingen'. In: ZfdA 138 (2009), S. 50-52.

Terrahe, Tina, Heinrich Steinhöwels Apollonius. Edition und Studien. Berlin, Boston 2013 (Frühe Neuzeit 179).

Thüring von Ringoltingen, Melusine (1456). Nach dem Erstdruck Basel: Richel um 1473/1474 hg. v. André Schnyder in Verb. mit Ursula Rautenberg. Bd. II, Kommentar und Aufsätze. Wiesbaden 2006.

Welz, Dieter, 'Der große Alexander’. In: ${ }^{2}$ VL 3 (1981), Sp. 281.

Wolff, Eva, 'Die Sprache der Bilder. Bild-Erzählung in den Handschriften der Romane der Elisabeth von Nassau-Saarbrücken'. In: Zwischen Deutschland und Frankreich. Elisabeth von Lothringen, Gräfin von Nassau-Saarbrücken. Hg. v. Wolfgang Haubrichs und HansWalter Herrmann, unter Mitw. v. Gerhard Sauder. St. Ingbert 2002, S. 591-622.

Worstbrock, Franz Josef, 'Sieder, Johann'. In: VL Deutscher Humanismus 2 (2013), Sp. 896-902.

Zapf, Volker, 'Meister Wichwolt'. In: Deutsches Literatur-Lexikon. Das Mittelalter, Bd. 3: Reiseberichte und Geschichtsschreibung. Hg. v. Wolfgang Achnitz. Berlin, Boston 2011, Sp. 510-514.

Zapf, Volker, 'Antonius von Pforr'. In: Deutsches Literatur-Lexikon: Das Mittelalter, Bd. 5: Epik. Hg. v. Wolfgang Achnitz. Berlin, Boston 2013, Sp. 1839-1844.

Zapf, Volker, 'Buch von Troja nach Guido de Columnis'. In: Deutsches Literatur-Lexikon: Das Mittelalter, Bd. 5: Epik. Hg. v. Wolfgang Achnitz. Berlin, Boston 2013, Sp. 1275-1277.

Zapf, Volker, 'Cleomades'. In: Deutsches Literatur-Lexikon: Das Mittelalter, Bd. 5: Epik. Hg. v. Wolfgang Achnitz. Berlin, Boston 2013, Sp. 1573-1575.

Zapf, Volker, 'Elsässisches Trojabuch'. In: Deutsches Literatur-Lexikon: Das Mittelalter, Bd. 5: Epik. Hg. v. Wolfgang Achnitz. Berlin, Boston 2013, Sp. 1143-1145.

Zapf, Volker, 'Herzog Ernst'. In: Deutsches Literatur-Lexikon: Das Mittelalter, Bd. 5: Epik. Hg. v. Wolfgang Achnitz. Berlin, Boston 2013, Sp. 149-165.

Zapf, Volker, 'Magelone'. In: Deutsches Literatur-Lexikon: Das Mittelalter, Bd. 5: Epik. Hg. v. Wolfgang Achnitz. Berlin, Boston 2013, Sp. 1881-1884.

Zapf, Volker, 'Oswald'. In: Deutsches Literatur-Lexikon: Das Mittelalter, Bd. 5: Epik. Hg. v. Wolfgang Achnitz. Berlin, Boston 2013, Sp. 1260. 
Zapf, Volker, 'Püterich von Reichertshausen'. In: Deutsches Literatur-Lexikon: Das Mittelalter, Bd. 5: Epik. Hg. v. Wolfgang Achnitz. Berlin, Boston 2013, Sp. 1669-1669.

Zapf, Volker, 'Warbeck'. In: Deutsches Literatur-Lexikon: Das Mittelalter, Bd. 5: Epik. Hg. v. Wolfgang Achnitz. Berlin, Boston 2013, Sp. 2050-2055.

Zapf, Volker, 'Wilhelm von Österreich'. In: Deutsches Literatur-Lexikon: Das Mittelalter, Bd. 5: Epik. Hg. v. Wolfgang Achnitz. Berlin, Boston 2013, Sp. 1785-1787.

Zimmermann, Karin, Die Codices Palatini germanici in der Universitätsbibliothek Heidelberg (Cod. Pal. Germ. 1-181). Wiesbaden 2003. 
